\title{
On the use of neural networks to evaluate performances of shell models for composites
}

\author{
Marco Petrolo* (i) and Erasmo Carrera
}

\author{
*Correspondence: \\ marco.petrolo@polito.it \\ MUL2 Group, Department of \\ Mechanical and Aerospace \\ Engineering, Politecnico di \\ Torino, Corso Duca degli Abruzzi \\ 24, 10129 Turin, Italy
}

\begin{abstract}
This paper presents a novel methodology to assess the accuracy of shell finite elements via neural networks. The proposed framework exploits the synergies among three well-established methods, namely, the Carrera Unified Formulation (CUF), the Finite Element Method (FE), and neural networks (NN). CUF generates the governing equations for any-order shell theories based on polynomial expansions over the thickness. FE provides numerical results feeding the NN for training. Multilayer NN have the generalized displacement variables, and the thickness ratio as inputs, and the target is the maximum transverse displacement. This work investigates the minimum requirements for the NN concerning the number of neurons and hidden layers, and the size of the training set. The results look promising as the NN requires a fraction of FE analyses for training, can evaluate the accuracy of any-order model, and can incorporate physical features, e.g., the thickness ratio, that drive the complexity of the mathematical model. In other words, NN can trigger fast informed decision-making on the structural model to use and the influence of design parameters without the need of modifying, rebuild, or rerun an FE model.
\end{abstract}

Keywords: Shell, Composites, Finite elements Neural networks, Best Theory Diagram

\section{Introduction}

Shell finite elements (FE) are standard options to model two-dimensional (2D) curved structures. In commercial codes, shell FE have the assumptions of the classical theories [1-3] leading to up to six degrees of freedom (DOF) per node. Such assumptions may be too restrictive in the case of composite structures in which the high transverse deformability and the transverse anisotropy require the proper modeling of shear and normal transverse stresses, and variations of the displacement field at the interface between two layers with different mechanical properties, i.e., the Zig-Zag effect [4]. 3D FE can incorporate such effects but can lead to prohibitive computational costs due to severe aspect ratio constraints. 2D FE remain computationally more efficient and attractive and, over the years, many strategies emerged to extend their capabilities via, for instance, the use of higher-order polynomial thickness expansions leading to increasing DOF per node [5]. This paper presents a new methodology to assess shell FE for linear static analyses

(c) The Author(s) 2020. This article is licensed under a Creative Commons Attribution 4.0 International License, which permits use, sharing, adaptation, distribution and reproduction in any medium or format, as long as you give appropriate credit to the original author(s) and the source, provide a link to the Creative Commons licence, and indicate if changes were made. The images or other third party material in this article are included in the article's Creative Commons licence, unless indicated otherwise in a credit line to the material. If material is not included in the article's Creative Commons licence and your intended use is not permitted by statutory regulation or exceeds the permitted use, you will need to obtain permission directly from the copyright holder. To view a copy of this licence, visit http://creativecommons.org/licenses/by/4.0/. 
of composites, and the following literature survey focuses on this specific area. More comprehensive reviews are in [6-9].

Concerning the solution schemes, analytical and FE strategies are among the most used. Analytical models received a great deal of interest as they provide very useful exact solutions to, for instance, verify FE modelings. Such exact solutions can take into account the shear deformability [10-16] or directly provide 3D solutions [17-23]. Research on refined shell FE focused on higher-order models [24-26], the inclusion of transverse stretching and continuity [27-29], and the development of solid-shell elements [30-37]. Regardless of the solution scheme, the most important strategies to enhance the capabilities of shell models are either asymptotic or axiomatic. The former exploit asymptotic expansions of most relevant parameter, e.g., the thickness ratio, to build models with a priori known accuracy as compared to 3D models [38-41]. The latter, on the other hand, build models based on assumptions and, usually, less assumptions lead to more cumbersome models. The axiomatic way has various directories starting from the improvement of classical models [42-49]. As mentioned above, the proper modeling of the transverse behavior of composites is decisive as proved by the efforts of many researchers over the past few years. The focus is on improved modelings of the interlaminar stresses and throughthe-thickness continuity [50-55], shear correction factors [56], Zig-Zag models [57-61], Layer-Wise (LW) models [62-65], and mixed formulations [66-68] allowing for the a priori modeling of transverse stresses.

Another powerful approach is the Proper Generalized Decomposition (PGD) method $[69,70]$ in which the construction of the refined model and the solution of the problem take place simultaneously.

From the structural standpoint, the methodology in this paper adopts the Carrera Unified Formulation (CUF) allowing to obtain any-order shell theory without formal changes in the problem matrices $[4,71,72]$. One of the capabilities of CUF is the axiomatic/asymptotic method (AAM) $[73,74]$ to analyze the relevance of any generalized displacement variable. The systematic use of AAM leads to the definition of the Best Theory Diagram (BTD), i.e., a 2D plot to localize shell models with minimum DOF and maximum accuracies [75,76]. One of the aims of this paper is to reduce the computational costs to obtain BTD via neural networks (NN). Such networks are mathematical models inspired by biological nervous systems and composed of simple computational units interlinked by a system of connections [77] to learn through training via samples. In this paper, CUF FE provides the samples for the supervised learning of multilayer perceptrons to evaluate the accuracy of refined shell models avoiding FE matrices and analyses. The use of $\mathrm{NN}$ in structural and material simulation is increasing due to the superior computational efficiency [78-80]. Recent applications for composites concern the prediction of the elastic properties [81], buckling load [82,83], failure strength [84,85], natural frequencies [86-88], and geometry optimization [89].

In this paper, "Finite element formulation" section provides a brief theoretical description of CUF and its FE formulation. "Best Theory Diagram" section introduces the concept of BTD. "Neural networks and coding" section describes the use of NN to evaluate the accuracy of a shell model. Results and conclusions are in "Results" and "Conclusions" sections, respectively. 


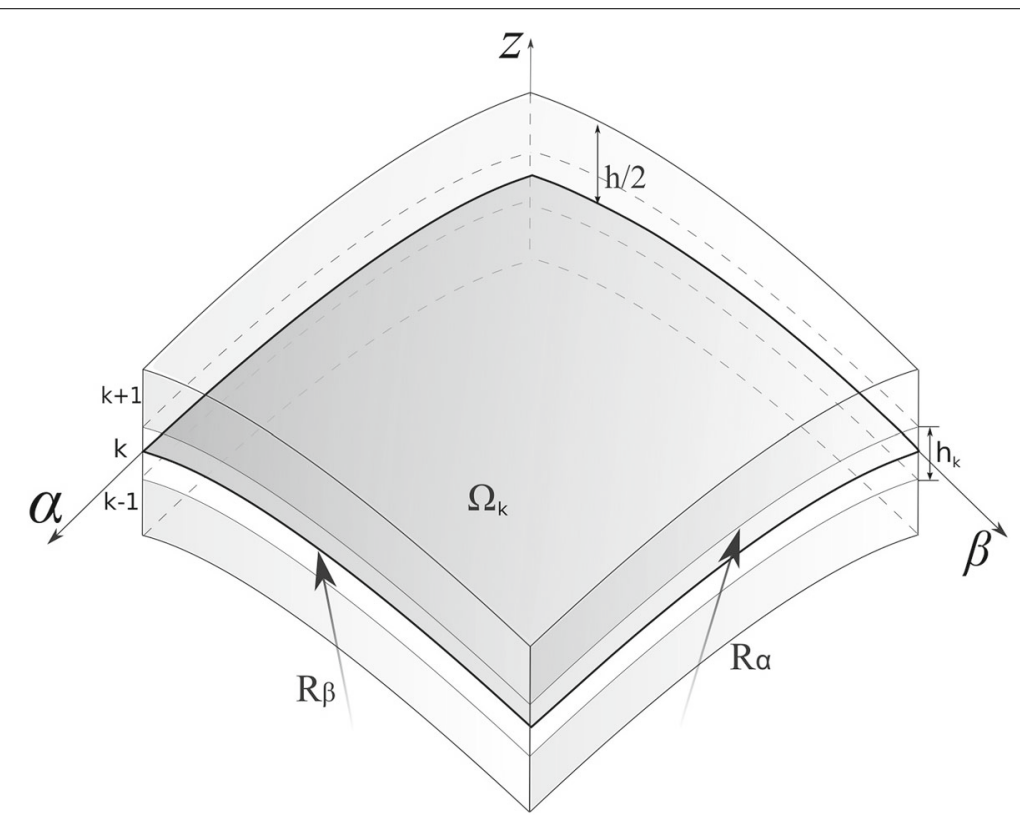

Fig. 1 Shell geometry

\section{Finite element formulation}

The CUF displacement field for a 2D model is

$$
\mathbf{u}(\alpha, \beta, z)=F_{\tau}(z) \mathbf{u}_{\tau}(\alpha, \beta) \quad \tau=1, \ldots, M
$$

The Einstein notation acts on $\tau . \mathbf{u}$ is the displacement vector, $\left(\mathbf{u}_{x} \quad \mathbf{u}_{y} \mathbf{u}_{z}\right)^{T} . \mathrm{F}_{\tau}$ are the thickness expansion functions. $\mathbf{u}_{\tau}$ is the vector of the generalized unknown displacements. $\mathrm{M}$ is the number of expansion terms. A fourth-order model, referred to as $N=4$, is

$$
\begin{aligned}
& u_{x}=u_{x_{1}}+z u_{x_{2}}+z^{2} u_{x_{3}}+z^{3} u_{x_{4}}+z^{4} u_{x_{5}} \\
& u_{y}=u_{y_{1}}+z u_{y_{2}}+z^{2} u_{y_{3}}+z^{3} u_{y_{4}}+z^{4} u_{y_{5}} \\
& u_{z}=u_{z_{1}}+z u_{z_{2}}+z^{2} u_{z_{3}}+z^{3} u_{z_{4}}+z^{4} u_{z_{5}}
\end{aligned}
$$

and has 15 nodal DOF. The order and type of expansion is a free parameter; thus, the theory of structure is an input of the analysis. The metric coefficients $\mathrm{H}_{\alpha}^{k}, \mathrm{H}_{\beta}^{k}$ and $\mathrm{H}_{z}^{k}$ of the kth layer are

$$
H_{\alpha}^{k}=A^{k}\left(1+z_{k} / R_{\alpha}^{k}\right), \quad H_{\beta}^{k}=B^{k}\left(1+z_{k} / R_{\beta}^{k}\right), \quad H_{z}^{k}=1
$$

$\mathrm{R}_{\alpha}^{k}$ and $\mathrm{R}_{\beta}^{k}$ are the principal radii of the middle surface of the kth layer, $\mathrm{A}^{k}$ and $\mathrm{B}^{k}$ the coefficients of the first fundamental form of $\Omega_{k}$, see Fig. 1. This paper focused only on shells with constant radii of curvature with $\mathrm{A}^{k}=\mathrm{B}^{k}=1$. The geometrical relations are

$$
\begin{aligned}
& \boldsymbol{\epsilon}_{p}^{k}=\left\{\epsilon_{\alpha \alpha}^{k}, \epsilon_{\beta \beta}^{k}, \epsilon_{\alpha \beta}^{k}\right\}^{T}=\left(\boldsymbol{D}_{p}^{k}+\boldsymbol{A}_{p}^{k}\right) \boldsymbol{u}^{k} \\
& \boldsymbol{\epsilon}_{n}^{k}=\left\{\epsilon_{\alpha z}^{k}, \epsilon_{\beta z}^{k}, \epsilon_{z z}^{k}\right\}^{T}=\left(\boldsymbol{D}_{n \Omega}^{k}+\boldsymbol{D}_{n z}^{k}-\boldsymbol{A}_{n}^{k}\right) \boldsymbol{u}^{k}
\end{aligned}
$$


where

$$
\begin{aligned}
& \boldsymbol{D}_{p}^{k}=\left[\begin{array}{ccc}
\frac{\partial_{\alpha}}{H_{\alpha}^{k}} & 0 & 0 \\
0 & \frac{\partial_{\beta}}{H_{\beta}^{k}} & 0 \\
\frac{\partial_{\beta}}{H_{\beta}^{k}} & \frac{\partial_{\alpha}}{H_{\alpha}^{k}} & 0
\end{array}\right] \boldsymbol{D}_{n \Omega}^{k}=\left[\begin{array}{ccc}
0 & 0 & \frac{\partial_{\alpha}}{H_{\alpha}^{k}} \\
0 & 0 & \frac{\partial_{\beta}^{k}}{H_{\beta}^{k}} \\
0 & 0 & 0
\end{array}\right] \quad D_{n z}^{k}=\left[\begin{array}{lll}
\partial_{z} & 0 & 0 \\
0 & \partial_{z} & 0 \\
0 & 0 & \partial_{z}
\end{array}\right] \\
& \boldsymbol{A}_{p}^{k}=\left[\begin{array}{ccc}
0 & 0 & \frac{1}{H_{\alpha}^{k} R_{\alpha}^{k}} \\
0 & 0 & \frac{1}{H_{\beta}^{k} R_{\beta}^{k}} \\
0 & 0 & 0
\end{array}\right] \boldsymbol{A}_{n}^{k}=\left[\begin{array}{ccc}
\frac{1}{H_{\alpha}^{k} R_{\alpha}^{k}} & 0 & 0 \\
0 & \frac{1}{H_{\beta}^{k} R_{\beta}^{k}} & 0 \\
0 & 0 & 0
\end{array}\right]
\end{aligned}
$$

The stress-strain relations are

$$
\begin{aligned}
& \boldsymbol{\sigma}_{p}^{k}=\left\{\sigma_{\alpha \alpha}^{k}, \sigma_{\beta \beta}^{k}, \sigma_{\alpha \beta}^{k}\right\}^{T}=C_{p p}^{k} \boldsymbol{\epsilon}_{p}^{k}+C_{p n}^{k} \boldsymbol{\epsilon}_{n}^{k} \\
& \boldsymbol{\sigma}_{n}^{k}=\left\{\sigma_{\alpha z}^{k}, \sigma_{\beta z}^{k}, \sigma_{z z}^{k}\right\}^{T}=C_{n p}^{k} \boldsymbol{\epsilon}_{p}^{k}+C_{n n}^{k} \boldsymbol{\epsilon}_{n}^{k}
\end{aligned}
$$

where

$$
\begin{array}{llc}
C_{p p}^{k}=\left[\begin{array}{lll}
C_{11}^{k} & C_{12}^{k} & C_{16}^{k} \\
C_{12}^{k} & C_{22}^{k} & C_{26}^{k} \\
C_{16}^{k} & C_{26}^{k} & C_{66}^{k}
\end{array}\right] & C_{p n}^{k}=\left[\begin{array}{ccc}
0 & 0 & C_{13}^{k} \\
0 & 0 & C_{23}^{k} \\
0 & 0 & C_{36}^{k}
\end{array}\right] \\
C_{n p}^{k}=\left[\begin{array}{ccc}
0 & 0 & 0 \\
0 & 0 & 0 \\
C_{13}^{k} & C_{23}^{k} & C_{36}^{k}
\end{array}\right] & C_{n n}^{k}=\left[\begin{array}{ccc}
C_{55}^{k} & C_{45}^{k} & 0 \\
C_{45}^{k} & C_{44}^{k} & 0 \\
0 & 0 & C_{33}^{k}
\end{array}\right]
\end{array}
$$

The FE formulation uses a nine-node shell element based on the Mixed Interpolation of Tensorial Component (MITC) method [90]. The displacement vector becomes

$$
\delta \boldsymbol{u}_{s}=N_{j} \delta \boldsymbol{u}_{s j}, \quad \boldsymbol{u}_{\tau}=N_{i} \boldsymbol{u}_{\tau i} \quad i, j=1, \ldots, 9
$$

$\boldsymbol{u}_{\tau i}$ and $\delta \boldsymbol{u}_{s j}$ are the nodal displacement vector and its virtual variation, respectively. The strain expression becomes

$$
\begin{aligned}
\boldsymbol{\epsilon}_{p} & =F_{\tau}\left(\boldsymbol{D}_{p}+\boldsymbol{A}_{p}\right) N_{i} \boldsymbol{u}_{\tau i} \\
\boldsymbol{\epsilon}_{n} & =F_{\tau}\left(\boldsymbol{D}_{n \Omega}-\boldsymbol{A}_{n}\right) N_{i} \boldsymbol{u}_{\tau i}+F_{\tau, z} N_{i} \boldsymbol{u}_{\tau i}
\end{aligned}
$$

MITC contrasts the membrane and shear locking via a specific interpolation strategy for the strain components on the nine-node shell element, as follows:

$$
\begin{aligned}
\boldsymbol{\epsilon}_{p} & =\left[\begin{array}{c}
\epsilon_{\alpha \alpha} \\
\epsilon_{\beta \beta} \\
\epsilon_{\alpha \beta}
\end{array}\right]=\left[\begin{array}{ccc}
N_{m 1} & 0 & 0 \\
0 & N_{m 2} & 0 \\
0 & 0 & N_{m 3}
\end{array}\right]\left[\begin{array}{l}
\epsilon_{\alpha \alpha_{m 1}} \\
\epsilon_{\beta \beta_{m 2}} \\
\epsilon_{\alpha \beta_{m 3}}
\end{array}\right] \\
\boldsymbol{\epsilon}_{n} & =\left[\begin{array}{c}
\epsilon_{\alpha z} \\
\epsilon_{\beta z} \\
\epsilon_{z z}
\end{array}\right]=\left[\begin{array}{ccc}
N_{m 1} & 0 & 0 \\
0 & N_{m 2} & 0 \\
0 & 0 & 1
\end{array}\right]\left[\begin{array}{l}
\epsilon_{\alpha z_{m 1}} \\
\epsilon_{\beta z_{m 2}} \\
\epsilon_{z z_{m 3}}
\end{array}\right]
\end{aligned}
$$

Strains $\epsilon_{\alpha \alpha_{m 1}}, \epsilon_{\beta \beta_{m 2}}, \epsilon_{\alpha \beta_{m 3}}, \epsilon_{\alpha z_{m 1}}$, and $\epsilon_{\beta z_{m 2}}$ stem from 10 and

$$
\begin{aligned}
& N_{m 1}=\left[N_{A 1}, N_{B 1}, N_{C 1}, N_{D 1}, N_{E 1}, N_{F 1}\right] \\
& N_{m 2}=\left[N_{A 2}, N_{B 2}, N_{C 2}, N_{D 2}, N_{E 2}, N_{F 2}\right] \\
& N_{m 3}=\left[N_{P}, N_{Q}, N_{R}, N_{S}\right]
\end{aligned}
$$

Subscripts $\mathrm{m} 1, \mathrm{~m} 2$ and $\mathrm{m} 3$ indicate the point groups (A1,B1,C1,D1,E1,F1), (A2,B2,C2, D2,E2,F2), and (P,Q,R,S), respectively, see Fig. 2. Via Principle of Virtual Displacements (PVD) for the static analysis, the equilibrium equation reads

$$
\boldsymbol{k}_{\tau s i j}^{k} \boldsymbol{u}_{\tau i}^{k}=\boldsymbol{p}_{s j}^{k}
$$



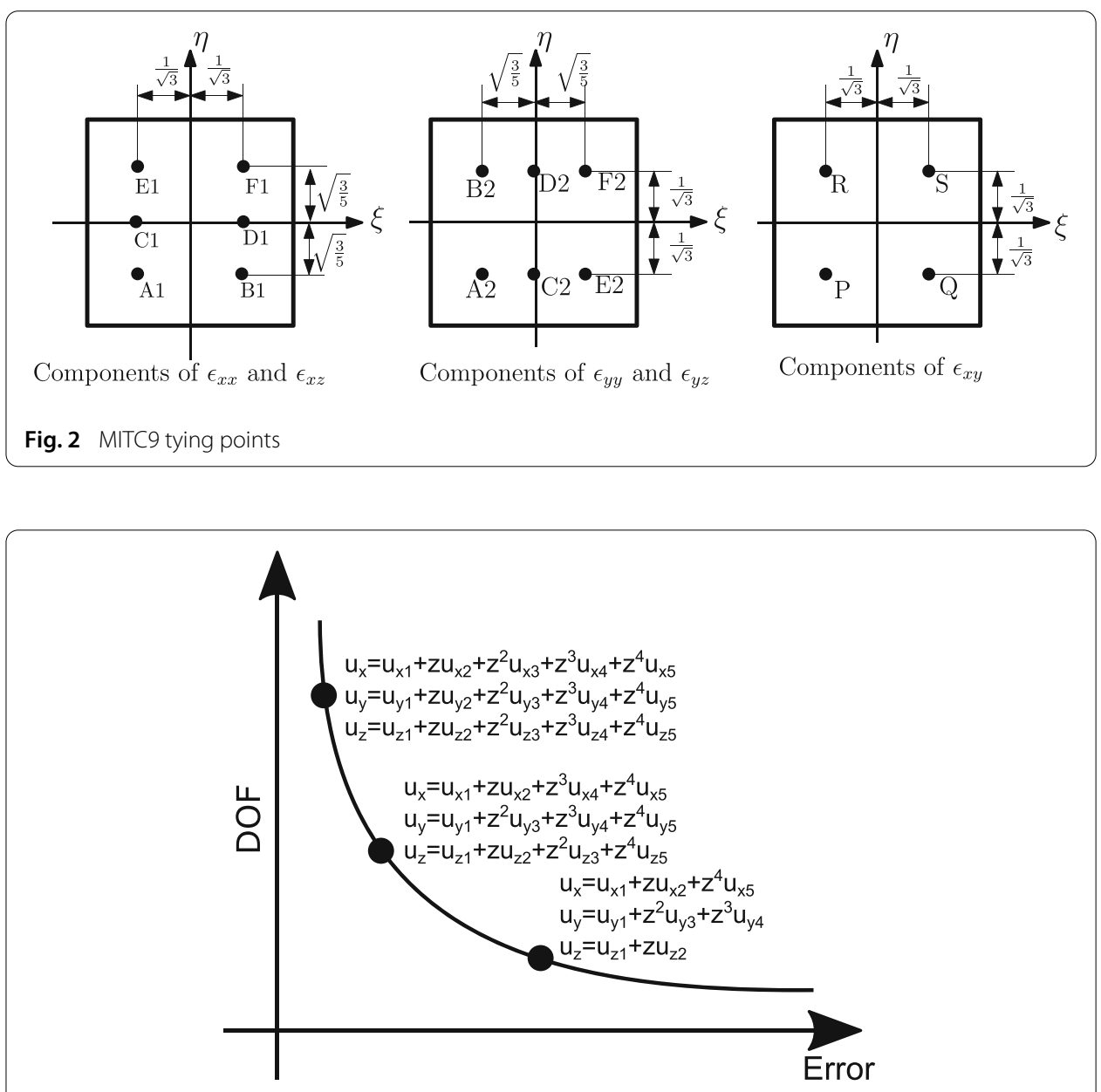

Fig. 3 Best Theory Diagram

The $3 \times 3$ matrix $\boldsymbol{k}_{\tau s i j}^{k}$ is the fundamental mechanical nucleus whose expression is independent of the order of the expansion. $\boldsymbol{p}_{s j}^{k}$ is the load vector. More details regarding the finite element formulation are in [72].

\section{Best Theory Diagram}

One of the CUF capabilities is the axiomatic/asymptotic method (AAM) to evaluate the relevance of generalized variables and the accuracy of structural theories [73,74]. The fourth-order, equivalent single layer shell model, is the reference model of this paper and all the theories evaluated stem from the combinations of the full fourth-order expansion, i.e., $2^{15}$ models. The CUF generates the governing equations for the theories considered. In particular, the CUF generates reduced models having combinations of the starting terms as generalized unknowns. Two parameters can identify a theory, namely, the number of active terms and the error or accuracy provided. The Best Theory Diagram (BTD) is the curve composed of all models providing the minimum error with the least number of variables, see Fig. 3. Given the accuracy, models with fewer variables than those on the BTD do not exist. Given the number of variables, models with better accuracy than those on the BTD do not exist. In this paper, the error refers to the maximum transverse 
Table 1 Examples of shell models assessed

\begin{tabular}{|c|c|c|c|c|c|c|c|c|c|c|c|c|c|c|c|c|}
\hline & DOF & $u_{\mathbf{x} 1}$ & $\mathbf{u}_{\mathbf{y} \mathbf{1}}$ & $\mathbf{u}_{\mathrm{z} 1}$ & $\mathbf{u}_{\mathbf{x} 2}$ & $\mathbf{u}_{\mathbf{y} 2}$ & $\mathbf{u}_{\mathrm{z} 2}$ & $\mathbf{u}_{\mathbf{x} 3}$ & $\mathbf{u}_{\mathbf{y} 3}$ & $\mathbf{u}_{\mathrm{z} 3}$ & $u_{x 4}$ & $u_{\mathbf{y} 4}$ & $\mathbf{u}_{\mathbf{z} 4}$ & $u_{x 5}$ & $u_{y 5}$ & $\mathbf{u}_{\mathbf{z} 5}$ \\
\hline$N=4$ & 15 & $\Delta$ & $\Delta$ & $\Delta$ & $\Delta$ & $\Delta$ & $\Delta$ & $\Delta$ & $\Delta$ & $\Delta$ & $\Delta$ & $\Delta$ & $\Delta$ & $\Delta$ & $\Delta$ & $\Delta$ \\
\hline TSDT & 9 & $\Delta$ & $\Delta$ & $\Delta$ & $\Delta$ & $\Delta$ & $\Delta$ & $\Delta$ & $\Delta$ & $\Delta$ & $\Delta$ & $\Delta$ & $\Delta$ & $\Delta$ & $\Delta$ & $\Delta$ \\
\hline PTD & 7 & $\Delta$ & $\Delta$ & $\Delta$ & $\Delta$ & $\boldsymbol{\Delta}$ & $\Delta$ & $\triangle$ & $\triangle$ & $\Delta$ & $\triangle$ & $\triangle$ & $\triangle$ & $\triangle$ & $\triangle$ & $\triangle$ \\
\hline FSDT & 5 & $\Delta$ & $\Delta$ & $\Delta$ & $\Delta$ & $\boldsymbol{\Delta}$ & $\Delta$ & $\Delta$ & $\Delta$ & $\Delta$ & $\Delta$ & $\Delta$ & $\Delta$ & $\Delta$ & $\triangle$ & $\Delta$ \\
\hline
\end{tabular}

displacement,

$$
\text { Error }=100 \times \frac{\left|u_{z}-u_{z}^{N=4}\right|}{\left|u_{z}^{N=4}\right|}
$$

The combined use of CUF and AAM allows the evaluation of the accuracy of any finite element, as shown in Table 1. Black and white triangles indicate active and inactive generalized displacement variables, respectively, and DOF the nodal degrees of freedom of the element. $\mathrm{N}=4$ is the full expansion of fourth-order. Other three models, well-known from literature, have incomplete expansions, namely,

- The First-Order Shear Deformation Theory (FSDT) with five DOF,

$$
\begin{aligned}
& u_{x}=u_{x_{1}}+z u_{x_{2}} \\
& u_{y}=u_{y_{1}}+z u_{y_{2}} \\
& u_{z}=u_{z_{1}}
\end{aligned}
$$

- A seven DOF model with parabolic transverse displacement, referred to as PTD,

$$
\begin{aligned}
& u_{x}=u_{x_{1}}+z u_{x_{2}} \\
& u_{y}=u_{y_{1}}+z u_{y_{2}} \\
& u_{z}=u_{z_{1}}+z u_{z_{2}}+z^{2} u_{z_{3}}
\end{aligned}
$$

- A nine DOF model with third-order in-plane displacements referred to as TSDT,

$$
\begin{aligned}
& u_{x}=u_{x_{1}}+z u_{x_{2}}+z^{2} u_{x_{3}}+z^{3} u_{x_{4}} \\
& u_{y}=u_{y_{1}}+z u_{y_{2}}+z^{2} u_{y_{3}}+z^{3} u_{y_{4}} \\
& u_{z}=u_{z_{1}}
\end{aligned}
$$

\section{Neural networks and coding}

CUF FE analyses generate inputs to train NN. In this paper, the inputs are the structural theories and the thickness ratio, and outputs are the maximum transverse displacements. Figure 4 shows the two ways adopted in this paper to build the BTD, i.e.,

- CUF generates the governing FE equations for all the shell theories stemming from subsets of the fourth-order expansions. Given that the expansion has 15 terms, overall, $2^{15} \mathrm{FE}$ shell models are available. For instance, FSDT is one of these models in which five terms are active $-\mathrm{u}_{x 1}, \mathrm{u}_{y 1}, \mathrm{u}_{z 1}, \mathrm{u}_{x 2}$, and $\mathrm{u}_{y 2}$-and ten inactive.

- The FE way runs $2^{15}$ static FE analyses and reports the error and number of active terms of each case in a $2 \mathrm{D}$ plot.

- The NN way runs one-tenth of the FE analyses and uses them for training. Then, the 2D plot stems from querying the trained NN with all $2^{15}$ shell models. 


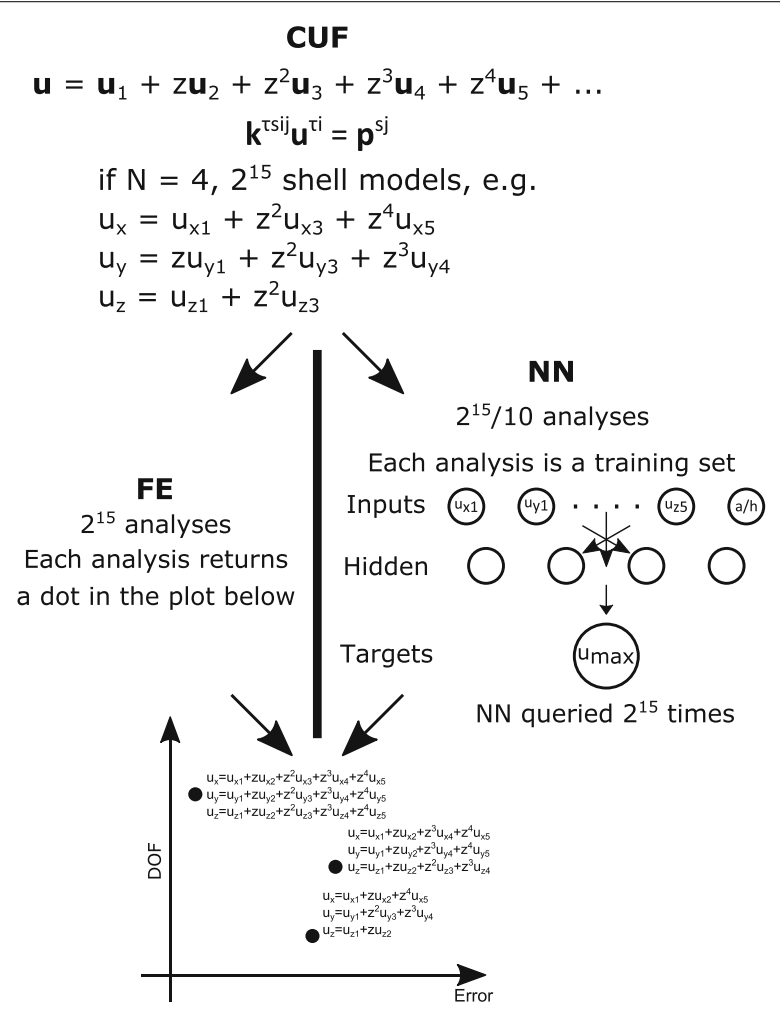

Fig. 4 CUF and NN framework

- If $\mathrm{a} / \mathrm{h}$ is a training variable, and, e.g., three $\mathrm{a} / \mathrm{h}$ values are available, the overall number of analyses is $3 \times 2^{15}$, and the query of the $\mathrm{NN}$ includes the shell model and the thickness ratio.

The aim is to build the BTD with less than $2^{15}$ analyses and avoid new FE analyses as the thickness ratio changes. In Fig. 4, the $\mathrm{NN}$ training set has $10 \%$ of all analyses as this is a typical value used in this paper. Also, the figure shows only one hidden layer, although more layers could be useful.

The NN configuration is a multilayer feed-forward with early stopping and mean squared error as the objective function. Each layer has ten neurons. This paper adopts Levenberg-Marquardt training functions [91]. The input coding is a vector with 16 elements, that is, all the fourth-order expansion generalized displacement variables and the thickness ratio. Each generalized variable is either ' 1 ' or ' 0 ' to indicate its active or inactive status. Each input has an associated output composed by a vector containing the error, Eq. 14. As an example, the following equation shows the coded input of a generic shell model with $\mathrm{h} / \mathrm{a}=0.1$ :

$$
\begin{aligned}
& u_{x}=u_{x_{1}}+z u_{x_{2}}+z^{4} u_{x_{5}}
\end{aligned}
$$

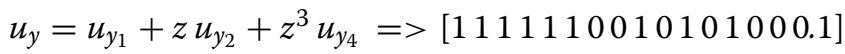

$$
\begin{aligned}
& u_{z}=u_{z_{1}}+z u_{z_{2}}+z^{2} u_{z_{3}}
\end{aligned}
$$

Table 2 presents the computational costs of the various processes involved in this paper. The cost normalization used the most expensive process as the reference. The number of layers was chosen via a convergence analysis as the adoption of more than one layer led to 
Table 2 Overciew of computational costs

\begin{tabular}{lll}
\hline Process & Analyses & Cost \\
\hline BTD via FE & 32768 linear static analyses & 1 \\
Data training generation & 3277 linear static analyses & 0.1 \\
Training of NN & $1-3$ layers with ten neurons & 0.01 \\
BTD via NN & $2^{15}$ queries to NN & 0.001 \\
\hline
\end{tabular}

Table 3 Comparison between NN and FE

\begin{tabular}{|c|c|}
\hline Structural theory and $\mathrm{a} / \mathrm{h}$ & $\begin{array}{l}u_{x}=u_{x_{1}}+z u_{x_{2}}+z^{4} u_{x_{5}} \\
u_{y}=u_{y_{1}}+z u_{y_{2}}+z^{3} u_{y_{4}} \\
u_{z}=u_{z_{1}}+z u_{z_{2}}+z^{2} u_{z_{3}} \\
a / h=10\end{array}$ \\
\hline Analysis & 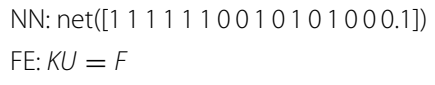 \\
\hline Output & $u_{z}$ \\
\hline
\end{tabular}

Table 4 0/90/0, $\bar{u}_{z}(z=0)=100 u_{z} E_{T} h^{3} /\left(\bar{p}_{z} a^{4}\right)$

\begin{tabular}{lll}
\hline Model & $\mathbf{R} / \mathbf{a}=\mathbf{5}$ & \\
\hline $3 \mathrm{D}[94]$ & - & 0.7325 \\
$\mathrm{LD} 4[93]$ & 0.1036 & 0.7325 \\
$\mathrm{~N}=4$ & 0.1036 & 0.6975 \\
$\mathrm{a} / \mathrm{h}$ & 100 & 10 \\
\hline
\end{tabular}

Table $50 / 90 / 0, a / h=100,10 \%$ training sets, one layer, influence of the number of neurons on some particular cases from Figs. 5, 6 and 7

\begin{tabular}{llllll}
\hline & Neurons & Training set & FSDT & PTD & TSDT \\
\hline FE & N/A & N/A & 0.424 & 0.060 & 0.365 \\
NN & 5 & $10 \%$ & 0.413 & 0.071 & 0.376 \\
NN & 10 & $5 \%$ & 0.460 & 0.096 & 0.416 \\
NN & 10 & $10 \%$ & 0.434 & 0.100 & 0.372 \\
\hline
\end{tabular}

negligible increments of the computational cost. For the type of NN adopted here, the use of 1-3 layers is a standard choice [91]. The data training generation used a random selection of the structural theories, and no significant variations in the results were observed between different set of randomly chosen training sets.

Table 3 shows an overview of the analyses employed to obtain the BTD. In all cases, the input is the structural theory. The capability of setting the theory as an input is a feature provided by CUF. As mentioned in previous sections, CUF allows one to handle the kinematics with no restrictions concerning the order and type of expansions adopted. Such a capability is decisive to obtain the BTD as a tool to verify the accuracy of any structural theory. In other words, via the BTD, the effect of the addition of a new generalized variable can be estimated. As the structural theory to be verified is set, the FE option requires the computation of the stiffness matrix and the solution of the linear static analysis. On the other hand, the trained NN can provide the output by encoding the structural model.

The use of NN has the aim to overcome two current limitations of BTD. First, the computational cost required can be very high as thousands of analyses are needed, and the complexity of the problem increases. Then, the evaluation of the BTD becomes even more challenging as various problem characteristics vary, e.g., boundary conditions or material 

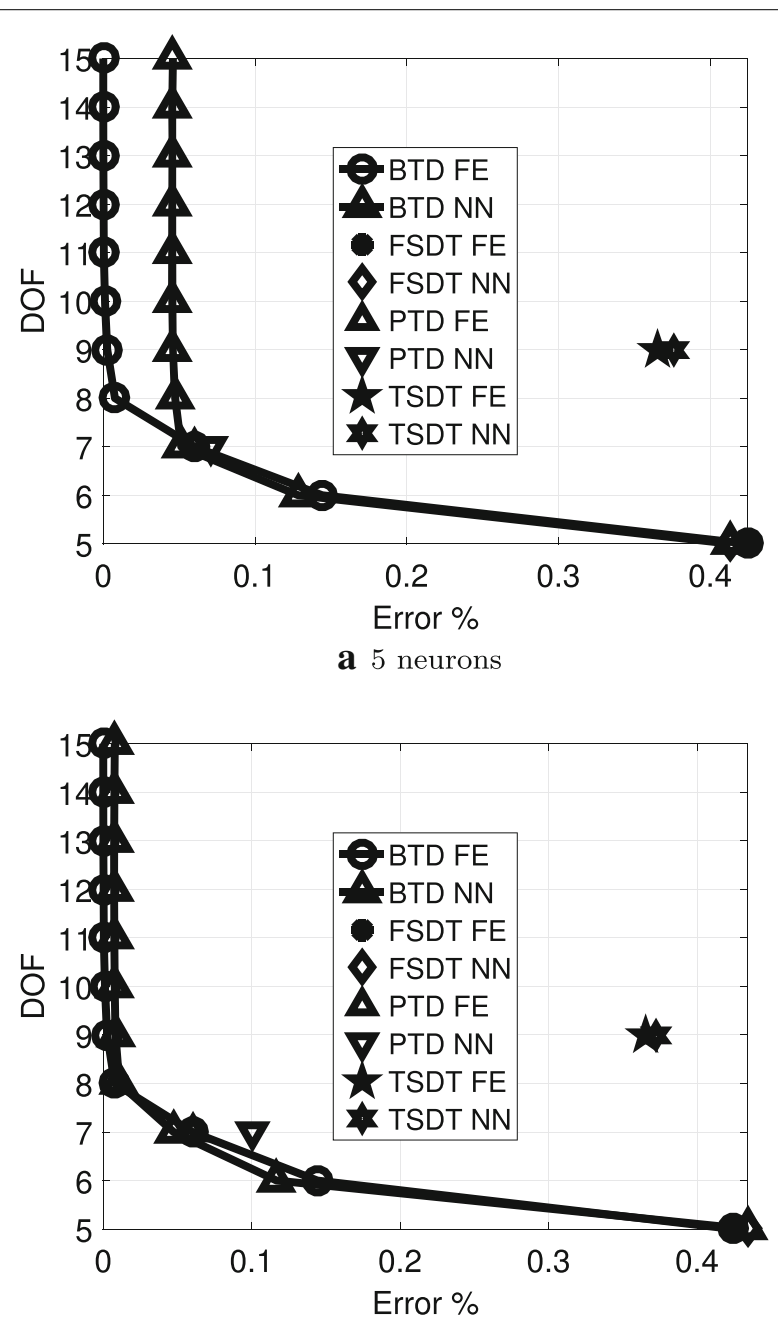

b 10 neurons

Fig. 5 FE and NN results for 0/90/0, a/h $=100,10 \%$ training sets, one layer, influence of the number of neurons

properties, and multiple outputs are considered, e.g., displacements and stresses. The use of NN may be a solution to both issues. This paper aims to address the first limitation and partially handling the second one. To address the second issue comprehensively, other NN architectures are needed, e.g., convolutional $\mathrm{NN}$ as they can manage high-dimensional input features with high efficiency [92].

\section{Results}

The numerical results focus on cases from [93]. The shell has $\mathrm{a}=\mathrm{b}, \mathrm{R}_{\alpha}=\mathrm{R}_{\beta}=\mathrm{R}$ and $\mathrm{R} / \mathrm{a}$ $=5$. The load is bi-sinusoidal and applied on the top surface, $\mathrm{p}_{z}=\hat{p}_{z} \sin (\pi \alpha / \mathrm{a}) \sin (\pi \beta / \mathrm{b})$. The material properties are $\mathrm{E}_{1} / \mathrm{E}_{2}=25, \mathrm{G}_{12} / \mathrm{E}_{2}=\mathrm{G}_{13} / \mathrm{E}_{2}=0.5, \mathrm{G}_{13} / \mathrm{E}_{2}=0.2, v=0.25$. The finite element model of a quarter of shell has a $4 \times 4$ mesh as this discretization provides sufficiently accurate results [93]. In all cases, the BTD vertical axis ranges from five to fifteen since models with four or less DOF provide very high errors and are not of practical interest. The numerical results stemmed from two methodologies as follows: 

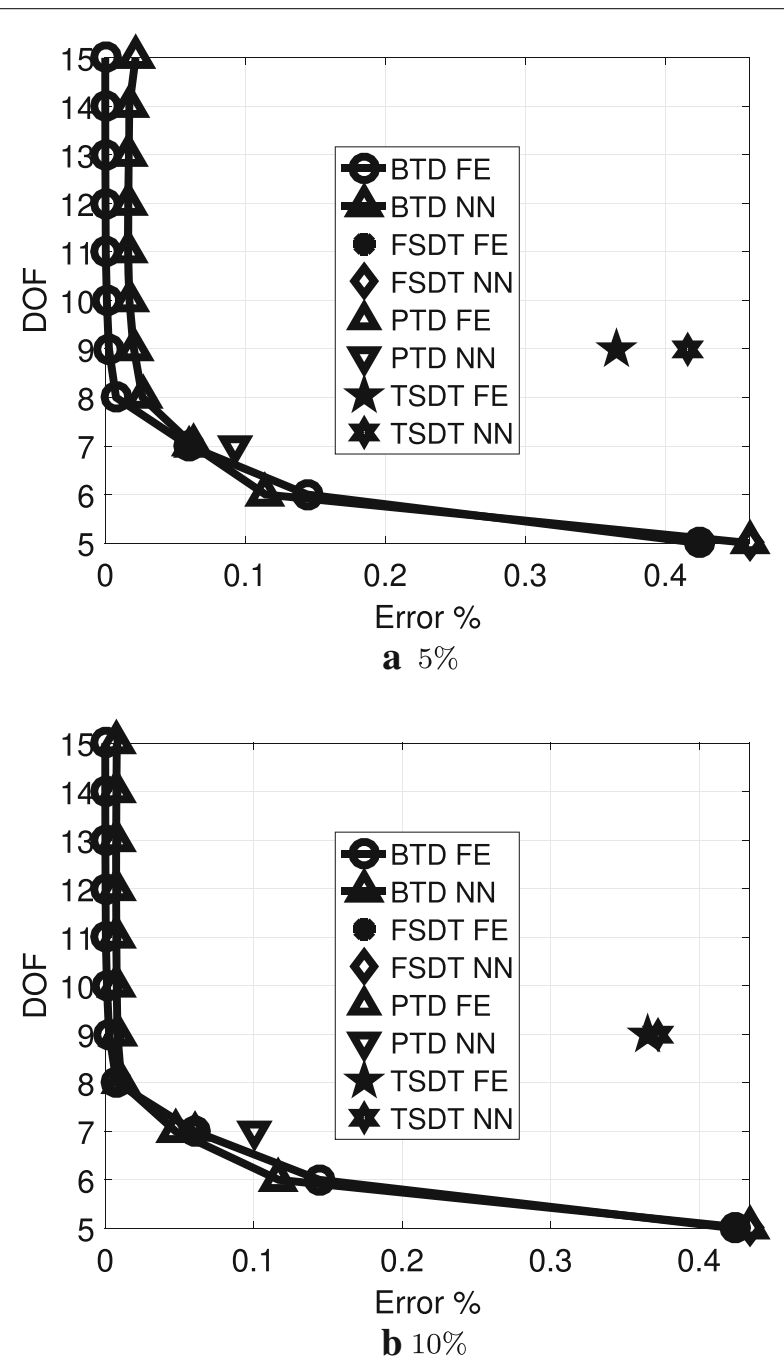

Fig. $6 \mathrm{FE}$ and NN results for $0 / 90 / 0, a / h=100$, one layer, influence of the number of the training set size

Table 6 0/90/0, influence of a/h on some particular cases from Figs. 8 and 9

\begin{tabular}{lllll}
\hline & $\mathbf{a} / \mathbf{h}$ & FSDT & PTD & TSDT \\
\hline $\mathrm{FE}$ & 50 & 1.192 & 0.495 & 0.695 \\
$\mathrm{NN}$ & 50 & 1.199 & 0.504 & 0.689 \\
$\mathrm{FE}$ & 10 & 12.12 & 11.50 & 0.571 \\
$\mathrm{NN}$ & 10 & 12.13 & 11.50 & 0.579 \\
\hline
\end{tabular}

- The finite element method, FE, required $2^{12}$ static analyses to build the BTD, i.e., one static analysis per each shell theory having a combination of 12 generalized variables. To lessen the computational cost, the three zeroth-order terms of the expansion are always active as, usually, their influence is very high.

- NN required $10 \%$ of $2^{12}$ to train, i.e., some 400 static analyses. Depending on the cases, the architecture of the network had one or three layers and ten neurons per layer. 

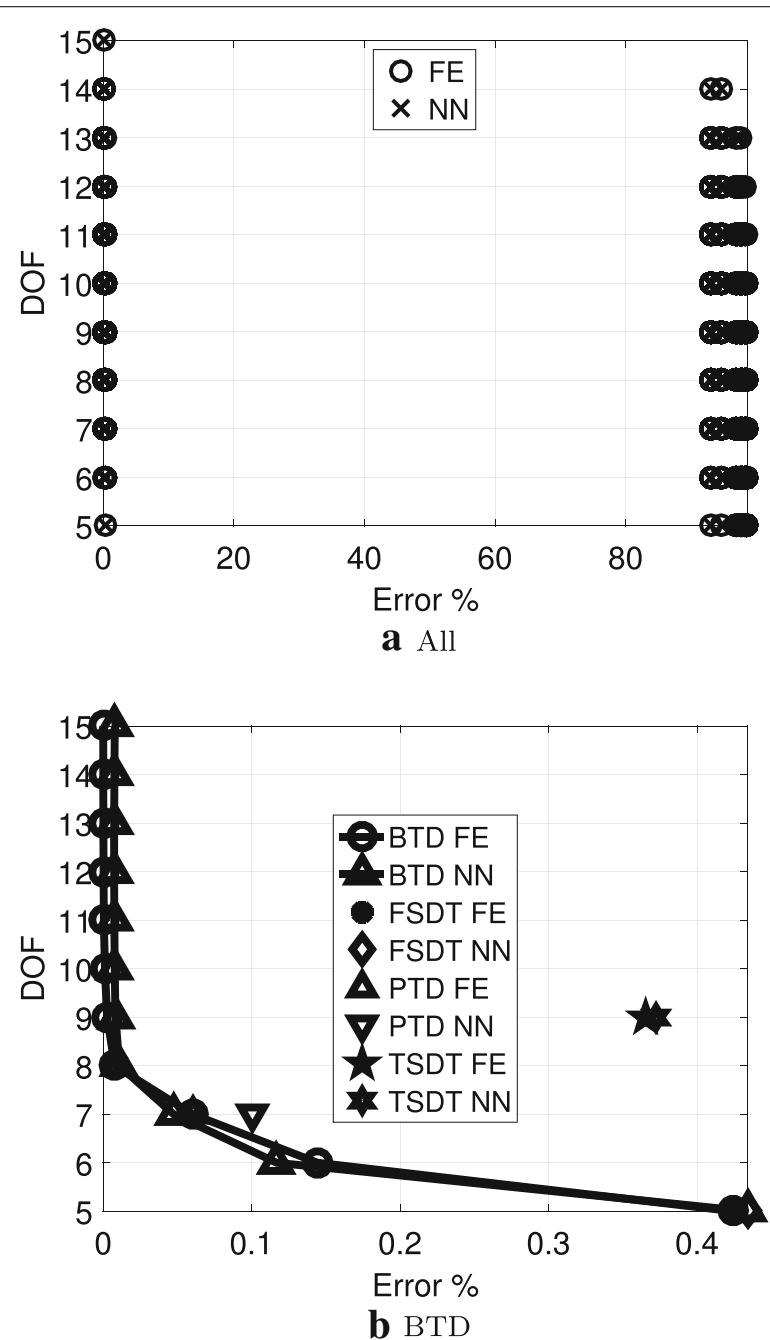

Fig. 7 FE and NN results for $0 / 90 / 0, a / h=100$, one layer with ten neurons

0/90/0

The first numerical case refers to a simply-supported shell with symmetric lamination. Table 4 shows the reference values of transverse displacements adopted to build the BTD. The current $\mathrm{N}=4$ model provides good accuracy, although, for thicker shells, the match with $3 \mathrm{D}$ solutions is not perfect. However, for the scope of the paper, its accuracy is sufficient.

First, the analysis concerned the choice of the network parameters. Figures 5 and 6 show the BTD from NN via 5 and 10 neurons and using $5 \%$ and $10 \%$ of the $2^{12}$ cases for training. Table 5 presents some particular cases focused on structural theories from the literature. The FE BTD serves as a benchmark. The results show that the use of 10 neurons and $10 \%$ of cases provides very good matches. The remaining analyses made use of such network architecture and focused on the effect of the thickness ratio on the BTD, given that, as seen in previous papers like [76], this is the most relevant parameter to determine the sets of most important generalized variables. Figure 7 shows the results for $\mathrm{a} / \mathrm{h}=100$ in which (a) reports the accuracy of all $2^{12}$ shell models as provided by the FE and by the 


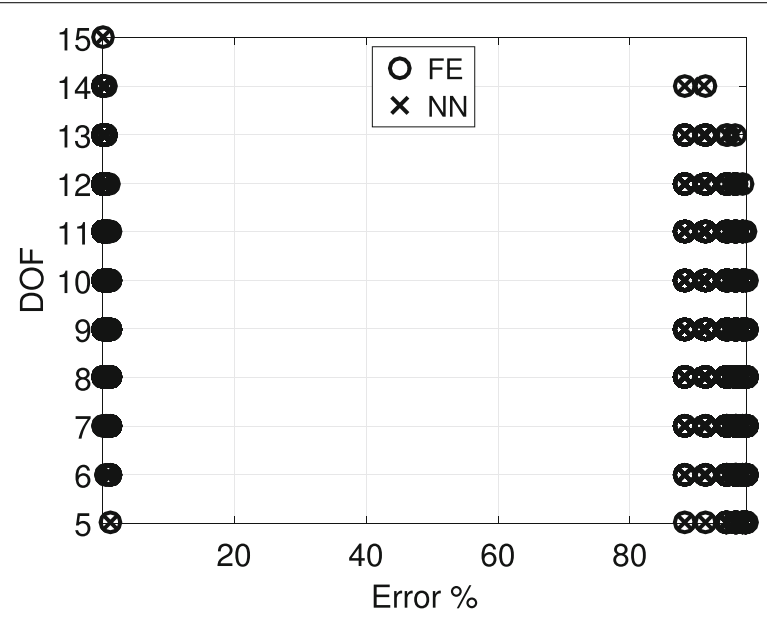

a All

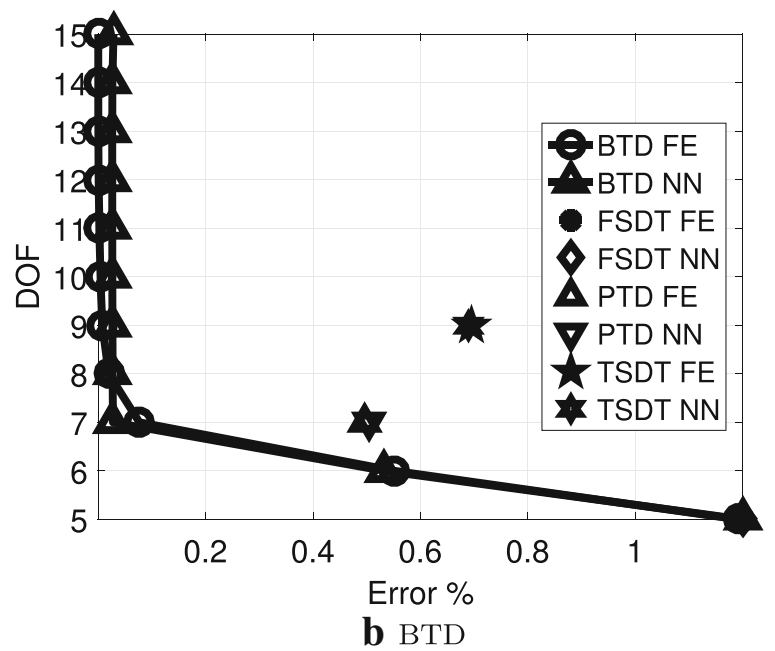

Fig. 8 FE and NN results for $0 / 90 / 0, a / h=50$, one layer with ten neurons

Table 7 BTD models, 0/90/0, $\mathrm{a} / \mathrm{h}=100$

\begin{tabular}{|c|c|c|c|c|c|c|c|c|c|c|c|c|c|c|c|}
\hline DOF & $u_{\mathbf{x} 1}$ & $\mathrm{u}_{\mathbf{y} 1}$ & $\mathbf{u}_{\mathrm{z} 1}$ & $u_{x 2}$ & $\mathbf{u}_{\mathrm{y} 2}$ & $\mathbf{u}_{\mathrm{z} 2}$ & $\mathbf{u}_{\mathbf{x} 3}$ & $\mathbf{u}_{\mathbf{y} 3}$ & $\mathbf{u}_{\mathrm{z} 3}$ & $\mathbf{u}_{\mathbf{x} 4}$ & $\mathrm{u}_{\mathrm{y} 4}$ & $\mathbf{u}_{\mathbf{z 4}}$ & $\mathrm{u}_{\mathrm{x} 5}$ & $\mathbf{u}_{\mathbf{y} 5}$ & $\mathbf{u}_{\mathrm{z} 5}$ \\
\hline 15 & $\Delta$ & $\Delta$ & $\Delta$ & $\Delta$ & $\Delta$ & $\Delta$ & $\Delta$ & $\Delta$ & $\boldsymbol{\Delta}$ & $\Delta$ & $\Delta$ & $\Delta$ & $\Delta$ & $\Delta$ & $\boldsymbol{\Delta}$ \\
\hline 14 & $\Delta$ & $\Delta$ & $\Delta$ & $\Delta$ & $\Delta$ & $\Delta$ & $\Delta$ & $\Delta$ & $\Delta$ & $\Delta$ & $\Delta$ & $\Delta$ & $\Delta$ & $\Delta$ & $\Delta$ \\
\hline 13 & $\Delta$ & $\Delta$ & $\Delta$ & $\Delta$ & $\Delta$ & $\Delta$ & $\Delta$ & $\Delta$ & $\Delta$ & $\Delta$ & $\Delta$ & $\Delta$ & $\Delta$ & $\Delta$ & $\Delta$ \\
\hline 12 & $\Delta$ & $\Delta$ & $\Delta$ & $\Delta$ & $\Delta$ & $\Delta$ & $\Delta$ & $\Delta$ & $\Delta$ & $\Delta$ & $\Delta$ & $\Delta$ & $\Delta$ & $\Delta$ & $\Delta$ \\
\hline 11 & $\Delta$ & $\Delta$ & $\Delta$ & $\Delta$ & $\Delta$ & $\Delta$ & $\Delta$ & $\Delta$ & $\Delta$ & $\Delta$ & $\Delta$ & $\Delta$ & $\Delta$ & $\Delta$ & $\Delta$ \\
\hline 10 & $\Delta$ & $\Delta$ & $\Delta$ & $\Delta$ & $\Delta$ & $\Delta$ & $\Delta$ & $\Delta$ & $\Delta$ & $\Delta$ & $\Delta$ & $\Delta$ & $\Delta$ & $\Delta$ & $\Delta$ \\
\hline 9 & $\Delta$ & $\Delta$ & $\Delta$ & $\Delta$ & $\Delta$ & $\Delta$ & $\Delta$ & $\Delta$ & $\boldsymbol{\Delta}$ & $\Delta$ & $\Delta$ & $\Delta$ & $\Delta$ & $\Delta$ & $\boldsymbol{\Delta}$ \\
\hline 8 & $\Delta$ & $\Delta$ & $\Delta$ & $\Delta$ & $\Delta$ & $\Delta$ & $\Delta$ & $\Delta$ & $\Delta$ & $\Delta$ & $\Delta$ & $\Delta$ & $\Delta$ & $\Delta$ & $\Delta$ \\
\hline 7 & $\Delta$ & $\Delta$ & $\Delta$ & $\Delta$ & $\Delta$ & $\Delta$ & $\Delta$ & $\Delta$ & $\Delta$ & $\Delta$ & $\Delta$ & $\Delta$ & $\Delta$ & $\Delta$ & $\Delta$ \\
\hline 6 & $\Delta$ & $\Delta$ & $\Delta$ & $\Delta$ & $\Delta$ & $\Delta$ & $\Delta$ & $\Delta$ & $\Delta$ & $\Delta$ & $\Delta$ & $\Delta$ & $\Delta$ & $\Delta$ & $\Delta$ \\
\hline 5 & $\Delta$ & $\Delta$ & $\Delta$ & $\Delta$ & $\Delta$ & $\Delta$ & $\Delta$ & $\Delta$ & $\Delta$ & $\Delta$ & $\Delta$ & $\Delta$ & & $\Delta$ & $\Delta$ \\
\hline & \multicolumn{3}{|c|}{$\mathrm{RF}_{0}=1.00$} & \multicolumn{3}{|c|}{$\mathrm{RF}_{1}=0.88$} & \multicolumn{3}{|c|}{$\mathrm{RF}_{2}=0.52$} & \multicolumn{3}{|c|}{$\mathrm{RF}_{3}=0.48$} & \multicolumn{3}{|c|}{$\mathrm{RF}_{4}=0.45$} \\
\hline
\end{tabular}




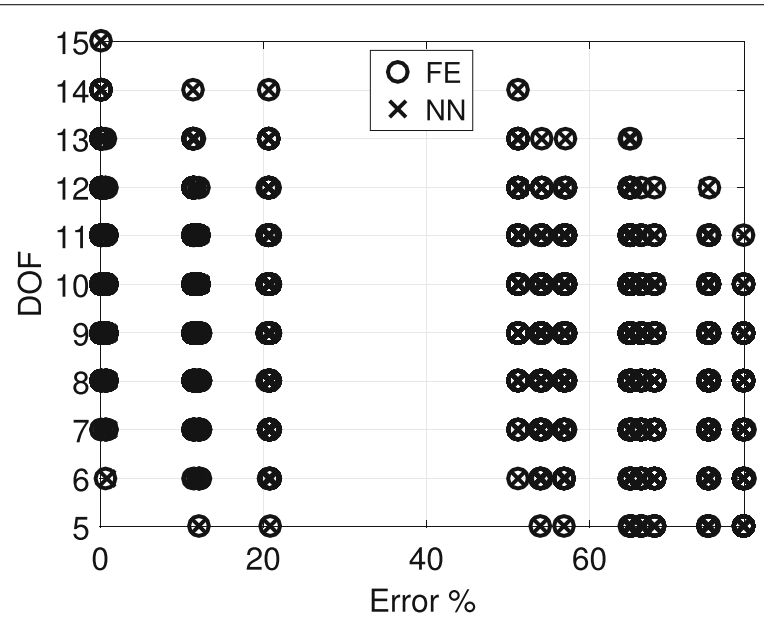

a All

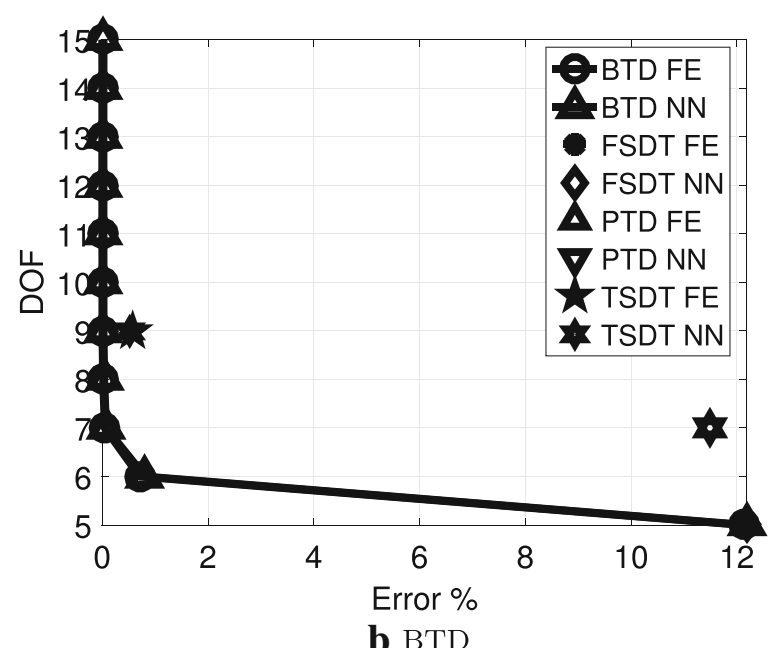

Fig. $9 \mathrm{FE}$ and $\mathrm{NN}$ results for $\mathrm{0} / 90 / 0, \mathrm{a} / \mathrm{h}=10$, one layer with ten neurons

Table 8 BTD models, $0 / 90 / 0, a / h=50$

\begin{tabular}{|c|c|c|c|c|c|c|c|c|c|c|c|c|c|c|c|}
\hline DOF & $\mathbf{u}_{x 1}$ & $\mathbf{u}_{\mathbf{y} 1}$ & $\mathbf{u}_{\mathrm{z} 1}$ & $u_{x 2}$ & $\mathrm{u}_{\mathrm{y} 2}$ & $\mathbf{u}_{\mathrm{z} 2}$ & $\mathbf{u}_{\mathbf{x} 3}$ & $\mathbf{u}_{\mathbf{y} 3}$ & $\mathbf{u}_{\mathrm{z} 3}$ & $u_{\times 4}$ & $\mathbf{u}_{\mathbf{y} 4}$ & $\mathbf{u}_{\mathbf{z} 4}$ & $u_{x 5}$ & $u_{y 5}$ & $\mathbf{u}_{\mathrm{z} 5}$ \\
\hline 15 & $\Delta$ & $\Delta$ & $\Delta$ & $\Delta$ & $\Delta$ & $\Delta$ & $\Delta$ & $\Delta$ & $\Delta$ & $\Delta$ & $\Delta$ & $\Delta$ & $\Delta$ & $\Delta$ & $\Delta$ \\
\hline 14 & $\Delta$ & $\Delta$ & $\Delta$ & $\Delta$ & $\Delta$ & $\Delta$ & $\Delta$ & $\Delta$ & $\Delta$ & $\Delta$ & $\Delta$ & $\Delta$ & $\Delta$ & $\Delta$ & $\Delta$ \\
\hline 13 & $\Delta$ & $\Delta$ & $\Delta$ & $\Delta$ & $\Delta$ & $\Delta$ & $\Delta$ & $\Delta$ & $\Delta$ & $\Delta$ & $\Delta$ & $\Delta$ & $\Delta$ & $\Delta$ & $\Delta$ \\
\hline 12 & $\Delta$ & $\Delta$ & $\Delta$ & $\Delta$ & $\Delta$ & $\Delta$ & $\Delta$ & $\Delta$ & $\Delta$ & $\Delta$ & $\Delta$ & $\Delta$ & $\Delta$ & $\Delta$ & $\Delta$ \\
\hline 11 & $\Delta$ & $\Delta$ & $\Delta$ & $\Delta$ & $\Delta$ & $\Delta$ & $\Delta$ & $\Delta$ & $\Delta$ & $\Delta$ & $\Delta$ & $\Delta$ & $\Delta$ & $\Delta$ & $\Delta$ \\
\hline 10 & $\Delta$ & $\Delta$ & $\Delta$ & $\Delta$ & $\Delta$ & $\Delta$ & $\Delta$ & $\Delta$ & $\Delta$ & $\Delta$ & $\Delta$ & $\Delta$ & $\Delta$ & $\Delta$ & $\Delta$ \\
\hline 9 & $\Delta$ & $\Delta$ & $\Delta$ & $\Delta$ & $\Delta$ & $\Delta$ & $\Delta$ & $\Delta$ & $\Delta$ & $\Delta$ & $\Delta$ & $\Delta$ & $\Delta$ & $\Delta$ & $\Delta$ \\
\hline 8 & $\Delta$ & $\Delta$ & $\Delta$ & $\Delta$ & $\Delta$ & $\Delta$ & $\Delta$ & $\Delta$ & $\Delta$ & $\Delta$ & $\Delta$ & $\Delta$ & $\Delta$ & $\Delta$ & $\Delta$ \\
\hline 7 & $\Delta$ & $\Delta$ & $\Delta$ & $\Delta$ & $\Delta$ & $\Delta$ & $\Delta$ & $\Delta$ & $\Delta$ & $\Delta$ & $\Delta$ & $\Delta$ & $\Delta$ & $\Delta$ & $\Delta$ \\
\hline 6 & $\Delta$ & $\Delta$ & $\Delta$ & $\Delta$ & $\Delta$ & $\Delta$ & $\Delta$ & $\Delta$ & $\Delta$ & $\Delta$ & $\Delta$ & $\Delta$ & $\Delta$ & $\Delta$ & $\Delta$ \\
\hline 5 & $\Delta$ & $\Delta$ & $\Delta$ & $\Delta$ & $\Delta$ & $\Delta$ & $\Delta$ & $\Delta$ & $\Delta$ & $\Delta$ & $\Delta$ & $\Delta$ & $\Delta$ & $\Delta$ & $\Delta$ \\
\hline & \multicolumn{3}{|c|}{$\mathrm{RF}_{0}=1.00$} & \multicolumn{3}{|c|}{$\mathrm{RF}_{1}=0.91$} & \multicolumn{3}{|c|}{$\mathrm{RF}_{2}=0.55$} & \multicolumn{3}{|c|}{$\mathrm{RF}_{3}=0.42$} & \multicolumn{3}{|c|}{$\mathrm{RF}_{4}=0.46$} \\
\hline
\end{tabular}


Table 9 BTD models, $0 / 90 / 0, a / h=10$

\begin{tabular}{|c|c|c|c|c|c|c|c|c|c|c|c|c|c|c|c|}
\hline OFD & $u_{\mathbf{x} 1}$ & $\mathbf{u}_{\mathbf{y} 1}$ & $\mathbf{u}_{\mathrm{z} 1}$ & $u_{x 2}$ & $\mathrm{u}_{\mathrm{y} 2}$ & $\mathbf{u}_{\mathrm{z} 2}$ & $\mathbf{u}_{\mathbf{x} 3}$ & $\mathbf{u}_{\mathbf{y} 3}$ & $\mathbf{u}_{\mathrm{z} 3}$ & $\mathbf{u}_{\mathbf{x} 4}$ & $\mathbf{u}_{\mathbf{y} 4}$ & $\mathbf{u}_{\mathbf{z} 4}$ & $\mathbf{u}_{\mathbf{x} 5}$ & $\mathbf{u}_{\mathbf{y} 5}$ & $\mathbf{u}_{\mathrm{z} 5}$ \\
\hline 15 & $\Delta$ & $\Delta$ & $\Delta$ & $\Delta$ & $\Delta$ & $\Delta$ & $\boldsymbol{\Delta}$ & $\Delta$ & $\Delta$ & $\Delta$ & $\Delta$ & $\Delta$ & $\Delta$ & $\Delta$ & $\Delta$ \\
\hline 14 & $\Delta$ & $\Delta$ & $\Delta$ & $\Delta$ & $\Delta$ & $\Delta$ & $\Delta$ & $\Delta$ & $\Delta$ & $\Delta$ & $\Delta$ & $\Delta$ & $\Delta$ & $\Delta$ & $\Delta$ \\
\hline 13 & $\Delta$ & $\Delta$ & $\Delta$ & $\Delta$ & $\Delta$ & $\Delta$ & $\Delta$ & $\Delta$ & $\Delta$ & $\Delta$ & $\Delta$ & $\Delta$ & $\Delta$ & $\Delta$ & $\Delta$ \\
\hline 12 & $\Delta$ & $\Delta$ & $\Delta$ & $\Delta$ & $\Delta$ & $\Delta$ & $\Delta$ & $\Delta$ & $\boldsymbol{\Delta}$ & $\Delta$ & $\Delta$ & $\Delta$ & $\Delta$ & $\boldsymbol{\Delta}$ & $\Delta$ \\
\hline 11 & $\Delta$ & $\Delta$ & $\Delta$ & $\Delta$ & $\Delta$ & $\Delta$ & $\Delta$ & $\Delta$ & $\Delta$ & $\Delta$ & $\Delta$ & $\Delta$ & $\Delta$ & $\Delta$ & $\Delta$ \\
\hline 10 & $\Delta$ & $\Delta$ & $\Delta$ & $\Delta$ & $\Delta$ & $\Delta$ & $\Delta$ & $\Delta$ & $\Delta$ & $\Delta$ & $\Delta$ & $\Delta$ & $\Delta$ & $\Delta$ & $\Delta$ \\
\hline 9 & $\Delta$ & $\Delta$ & $\Delta$ & $\Delta$ & $\Delta$ & $\Delta$ & $\Delta$ & $\Delta$ & $\Delta$ & $\Delta$ & $\Delta$ & $\Delta$ & $\Delta$ & $\Delta$ & $\Delta$ \\
\hline 8 & $\Delta$ & $\Delta$ & $\Delta$ & $\Delta$ & $\Delta$ & $\Delta$ & $\Delta$ & $\Delta$ & $\Delta$ & $\Delta$ & $\Delta$ & $\Delta$ & $\Delta$ & $\Delta$ & $\Delta$ \\
\hline 7 & $\Delta$ & $\Delta$ & $\Delta$ & $\Delta$ & $\Delta$ & $\Delta$ & $\Delta$ & $\Delta$ & $\Delta$ & $\Delta$ & $\Delta$ & $\Delta$ & $\Delta$ & $\Delta$ & $\Delta$ \\
\hline 6 & $\Delta$ & $\Delta$ & $\Delta$ & $\Delta$ & $\Delta$ & $\Delta$ & $\Delta$ & $\Delta$ & $\Delta$ & $\Delta$ & $\Delta$ & $\Delta$ & $\Delta$ & $\Delta$ & $\Delta$ \\
\hline 5 & $\Delta$ & $\Delta$ & $\Delta$ & $\Delta$ & $\Delta$ & $\Delta$ & $\Delta$ & $\Delta$ & $\Delta$ & $\Delta$ & $\Delta$ & $\Delta$ & $\Delta$ & $\Delta$ & $\Delta$ \\
\hline & \multicolumn{3}{|c|}{$\mathrm{RF}_{0}=1.00$} & \multicolumn{3}{|c|}{$\mathrm{RF}_{1}=0.70$} & \multicolumn{3}{|c|}{$\mathrm{RF}_{2}=0.55$} & \multicolumn{3}{|c|}{$\mathrm{RF}_{3}=0.61$} & \multicolumn{3}{|c|}{$\mathrm{RF}_{4}=0.49$} \\
\hline
\end{tabular}

Table $100 / 90 / 0 / 90, \bar{u}_{z}(z=0)=100 u_{z} \mathrm{E}_{T} \mathrm{~h}^{3} /\left(\bar{p}_{z} a^{4}\right)$

\begin{tabular}{lll}
\hline Model & $\mathbf{R} / \mathbf{a}=\mathbf{5}$ & \\
\hline 3D [94] & - & 0.7408 \\
LD4 [93] & 0.1067 & 0.7408 \\
$\mathrm{~N}=4$ & 0.1067 & 0.7055 \\
a/h & 100 & 10 \\
\hline
\end{tabular}

Table $110 / 90 / 0 / 90$, influence of $\mathrm{a} / \mathrm{h}$ on some particular cases from Figs. 13, 14 and 15

\begin{tabular}{lrlll}
\hline & $\mathbf{a} / \mathbf{h}$ & FSDT & PTD & TSDT \\
\hline FE & 100 & 0.387 & 0.035 & 0.361 \\
NN & 100 & 0.390 & 0.063 & 0.363 \\
FE & 50 & 1.533 & 1.412 & 1.207 \\
NN & 50 & 1.554 & 1.424 & 1.226 \\
FE & 10 & 10.43 & 9.833 & 3.374 \\
NN & 10 & 10.37 & 10.49 & 3.560 \\
\hline
\end{tabular}

Table 12 BTD models, 0/90/0/90, $\mathrm{a} / \mathrm{h}=100$

\begin{tabular}{|c|c|c|c|c|c|c|c|c|c|c|c|c|c|c|c|}
\hline DOF & $u_{x 1}$ & $\mathrm{u}_{\mathrm{y} 1}$ & $\mathbf{u}_{\mathrm{z} 1}$ & $u_{x 2}$ & $u_{y_{2}}$ & $\mathbf{u}_{\mathrm{z} 2}$ & $u_{x 3}$ & $\mathrm{u}_{\mathrm{y} 3}$ & $\mathbf{u}_{\mathrm{z} 3}$ & $\mathrm{u}_{\mathrm{x} 4}$ & $\mathrm{u}_{\mathrm{y} 4}$ & $\mathbf{u}_{\mathrm{z} 4}$ & $u_{x 5}$ & $\mathrm{u}_{\mathrm{y} 5}$ & $\mathbf{u}_{\mathrm{z} 5}$ \\
\hline 15 & $\Delta$ & $\Delta$ & $\Delta$ & $\Delta$ & $\Delta$ & $\Delta$ & $\Delta$ & $\Delta$ & $\Delta$ & $\Delta$ & $\Delta$ & $\Delta$ & $\Delta$ & $\Delta$ & $\Delta$ \\
\hline 14 & $\Delta$ & $\Delta$ & $\Delta$ & $\Delta$ & $\Delta$ & $\Delta$ & $\Delta$ & $\Delta$ & $\Delta$ & $\Delta$ & $\Delta$ & $\Delta$ & $\Delta$ & $\Delta$ & $\Delta$ \\
\hline 13 & $\Delta$ & $\Delta$ & $\Delta$ & $\Delta$ & $\Delta$ & $\Delta$ & $\Delta$ & $\Delta$ & $\Delta$ & $\Delta$ & $\Delta$ & $\Delta$ & $\Delta$ & $\Delta$ & $\Delta$ \\
\hline 12 & $\Delta$ & $\Delta$ & $\Delta$ & $\Delta$ & $\Delta$ & $\Delta$ & $\Delta$ & $\Delta$ & $\Delta$ & $\Delta$ & $\Delta$ & $\Delta$ & $\Delta$ & $\Delta$ & $\Delta$ \\
\hline 11 & $\Delta$ & $\Delta$ & $\Delta$ & $\Delta$ & $\Delta$ & $\Delta$ & $\Delta$ & $\Delta$ & $\Delta$ & $\Delta$ & $\Delta$ & $\Delta$ & $\Delta$ & $\Delta$ & $\Delta$ \\
\hline 10 & $\Delta$ & $\Delta$ & $\Delta$ & $\Delta$ & $\Delta$ & $\Delta$ & $\Delta$ & $\Delta$ & $\Delta$ & $\Delta$ & $\Delta$ & $\Delta$ & $\Delta$ & $\Delta$ & $\Delta$ \\
\hline 9 & $\Delta$ & $\Delta$ & $\Delta$ & $\Delta$ & $\Delta$ & $\Delta$ & $\Delta$ & $\Delta$ & $\Delta$ & $\Delta$ & $\Delta$ & $\Delta$ & $\Delta$ & $\Delta$ & $\Delta$ \\
\hline 8 & $\Delta$ & $\Delta$ & $\Delta$ & $\Delta$ & $\Delta$ & $\Delta$ & $\Delta$ & $\Delta$ & $\Delta$ & $\Delta$ & $\Delta$ & $\Delta$ & $\Delta$ & $\Delta$ & $\Delta$ \\
\hline 7 & $\Delta$ & $\Delta$ & $\Delta$ & $\Delta$ & $\Delta$ & $\Delta$ & $\Delta$ & $\Delta$ & $\Delta$ & $\Delta$ & $\Delta$ & $\Delta$ & $\Delta$ & $\Delta$ & $\Delta$ \\
\hline 6 & $\Delta$ & $\Delta$ & $\Delta$ & $\Delta$ & $\Delta$ & $\Delta$ & $\Delta$ & $\Delta$ & $\Delta$ & $\Delta$ & $\Delta$ & $\Delta$ & $\Delta$ & $\Delta$ & $\Delta$ \\
\hline 5 & $\Delta$ & $\Delta$ & $\Delta$ & $\Delta$ & $\Delta$ & $\Delta$ & $\Delta$ & $\Delta$ & $\Delta$ & $\Delta$ & $\Delta$ & $\Delta$ & $\Delta$ & $\Delta$ & $\Delta$ \\
\hline & \multicolumn{3}{|c|}{$\mathrm{RF}_{0}=1.00$} & \multicolumn{3}{|c|}{$\mathrm{RF}_{1}=0.88$} & \multicolumn{3}{|c|}{$\mathrm{RF}_{2}=0.52$} & \multicolumn{3}{|c|}{$\mathrm{RF}_{3}=0.49$} & \multicolumn{3}{|c|}{$\mathrm{RF}_{4}=0.46$} \\
\hline
\end{tabular}


Table 13 BTD models, $0 / 90 / 0 / 90, a / h=50$

\begin{tabular}{|c|c|c|c|c|c|c|c|c|c|c|c|c|c|c|c|}
\hline DOF & $u_{\mathbf{x} 1}$ & $u_{\mathbf{y} 1}$ & $\mathbf{u}_{\mathrm{z} 1}$ & $u_{x 2}$ & $\mathrm{u}_{\mathrm{y} 2}$ & $\mathbf{u}_{\mathrm{z} 2}$ & $\mathbf{u}_{\mathbf{x} 3}$ & $\mathrm{u}_{\mathrm{y} 3}$ & $\mathbf{u}_{\mathrm{z} 3}$ & $u_{x 4}$ & $\mathrm{u}_{\mathrm{y} 4}$ & $\mathbf{u}_{\mathrm{z} 4}$ & $u_{x 5}$ & $u_{y 5}$ & $\mathbf{u}_{\mathrm{z} 5}$ \\
\hline 15 & $\Delta$ & $\Delta$ & $\Delta$ & $\Delta$ & $\Delta$ & $\Delta$ & $\Delta$ & $\Delta$ & $\Delta$ & $\Delta$ & $\Delta$ & $\Delta$ & $\Delta$ & $\Delta$ & $\Delta$ \\
\hline 14 & $\Delta$ & $\Delta$ & $\Delta$ & $\Delta$ & $\Delta$ & $\Delta$ & $\Delta$ & $\Delta$ & $\Delta$ & $\Delta$ & $\Delta$ & $\Delta$ & $\Delta$ & $\Delta$ & $\Delta$ \\
\hline 13 & $\Delta$ & $\Delta$ & $\Delta$ & $\Delta$ & $\Delta$ & $\Delta$ & $\Delta$ & $\Delta$ & $\Delta$ & $\Delta$ & $\Delta$ & $\Delta$ & $\Delta$ & $\Delta$ & $\Delta$ \\
\hline 12 & $\Delta$ & $\Delta$ & $\Delta$ & $\Delta$ & $\Delta$ & $\Delta$ & $\Delta$ & $\Delta$ & $\Delta$ & $\Delta$ & $\Delta$ & $\Delta$ & $\Delta$ & $\Delta$ & $\Delta$ \\
\hline 11 & $\Delta$ & $\Delta$ & $\Delta$ & $\Delta$ & $\Delta$ & $\Delta$ & $\Delta$ & $\Delta$ & $\Delta$ & $\Delta$ & $\Delta$ & $\Delta$ & $\Delta$ & $\Delta$ & $\Delta$ \\
\hline 10 & $\Delta$ & $\Delta$ & $\Delta$ & $\Delta$ & $\Delta$ & $\Delta$ & $\Delta$ & $\Delta$ & $\Delta$ & $\Delta$ & $\Delta$ & $\Delta$ & $\Delta$ & $\Delta$ & $\Delta$ \\
\hline 9 & $\Delta$ & $\Delta$ & $\Delta$ & $\Delta$ & $\Delta$ & $\Delta$ & $\Delta$ & $\Delta$ & $\Delta$ & $\Delta$ & $\Delta$ & $\Delta$ & $\Delta$ & $\Delta$ & $\Delta$ \\
\hline 8 & $\Delta$ & $\Delta$ & $\Delta$ & $\Delta$ & $\Delta$ & $\Delta$ & $\Delta$ & $\Delta$ & $\Delta$ & $\Delta$ & $\Delta$ & $\Delta$ & $\Delta$ & $\Delta$ & $\Delta$ \\
\hline 7 & $\Delta$ & $\Delta$ & $\Delta$ & $\Delta$ & $\Delta$ & $\Delta$ & $\Delta$ & $\Delta$ & $\Delta$ & $\Delta$ & $\Delta$ & $\Delta$ & $\Delta$ & $\Delta$ & $\Delta$ \\
\hline 6 & $\Delta$ & $\Delta$ & $\Delta$ & $\Delta$ & $\Delta$ & $\Delta$ & $\Delta$ & $\Delta$ & $\Delta$ & $\Delta$ & $\Delta$ & $\Delta$ & $\Delta$ & $\Delta$ & $\Delta$ \\
\hline 5 & $\Delta$ & $\Delta$ & $\Delta$ & $\Delta$ & $\Delta$ & $\Delta$ & $\Delta$ & $\Delta$ & $\Delta$ & $\Delta$ & $\Delta$ & $\Delta$ & $\Delta$ & $\Delta$ & $\Delta$ \\
\hline & \multicolumn{3}{|c|}{$\mathrm{RF}_{0}=1.00$} & \multicolumn{3}{|c|}{$\mathrm{RF}_{1}=0.73$} & \multicolumn{3}{|c|}{$\mathrm{RF}_{2}=0.64$} & \multicolumn{3}{|c|}{$\mathrm{RF}_{3}=0.55$} & \multicolumn{3}{|c|}{$\mathrm{RF}_{4}=0.42$} \\
\hline
\end{tabular}

Table 14 BTD models, 0/90/0/90, $\mathrm{a} / \mathrm{h}=10$

\begin{tabular}{|c|c|c|c|c|c|c|c|c|c|c|c|c|c|c|c|}
\hline OFD & $\mathbf{u}_{\mathbf{x} 1}$ & $u_{\mathbf{y} 1}$ & $\mathbf{u}_{\mathrm{z} 1}$ & $u_{x 2}$ & $u_{\mathrm{y} 2}$ & $\mathbf{u}_{\mathrm{z} 2}$ & $u_{x 3}$ & $\mathbf{u}_{\mathbf{y} 3}$ & $\mathbf{u}_{\mathrm{z} 3}$ & $\mathbf{u}_{\mathbf{x} 4}$ & $\mathbf{u}_{\mathrm{y} 4}$ & $\mathbf{u}_{\mathrm{z} 4}$ & $u_{x 5}$ & $u_{y 5}$ & $\mathbf{u}_{\mathrm{z} 5}$ \\
\hline 15 & $\Delta$ & $\Delta$ & $\Delta$ & $\Delta$ & $\Delta$ & $\Delta$ & $\Delta$ & $\Delta$ & $\Delta$ & $\Delta$ & $\Delta$ & $\Delta$ & $\Delta$ & $\Delta$ & $\Delta$ \\
\hline 14 & $\Delta$ & $\Delta$ & $\Delta$ & $\Delta$ & $\Delta$ & $\Delta$ & $\Delta$ & $\Delta$ & $\Delta$ & $\Delta$ & $\Delta$ & $\Delta$ & $\Delta$ & $\Delta$ & $\Delta$ \\
\hline 13 & $\Delta$ & $\Delta$ & $\Delta$ & $\Delta$ & $\Delta$ & $\triangle$ & $\Delta$ & $\Delta$ & $\Delta$ & $\Delta$ & $\Delta$ & $\triangle$ & $\Delta$ & $\Delta$ & $\Delta$ \\
\hline 12 & $\Delta$ & $\Delta$ & $\Delta$ & $\Delta$ & $\Delta$ & $\triangle$ & $\Delta$ & $\Delta$ & $\triangle$ & $\Delta$ & $\Delta$ & $\triangle$ & $\Delta$ & $\Delta$ & $\Delta$ \\
\hline 11 & $\Delta$ & $\boldsymbol{\Delta}$ & $\Delta$ & $\Delta$ & $\boldsymbol{\Delta}$ & $\Delta$ & $\Delta$ & $\Delta$ & $\Delta$ & $\Delta$ & $\Delta$ & $\Delta$ & $\Delta$ & $\Delta$ & $\Delta$ \\
\hline 10 & $\boldsymbol{\Delta}$ & $\boldsymbol{\Delta}$ & $\boldsymbol{\Delta}$ & $\Delta$ & $\Delta$ & $\Delta$ & $\boldsymbol{\Delta}$ & $\Delta$ & $\Delta$ & $\Delta$ & $\Delta$ & $\Delta$ & $\Delta$ & $\Delta$ & $\Delta$ \\
\hline 9 & $\Delta$ & $\Delta$ & $\Delta$ & $\Delta$ & $\Delta$ & $\triangle$ & $\Delta$ & $\triangle$ & $\triangle$ & $\Delta$ & $\Delta$ & $\triangle$ & $\Delta$ & $\triangle$ & $\triangle$ \\
\hline 8 & $\Delta$ & $\Delta$ & $\Delta$ & $\Delta$ & $\boldsymbol{\Delta}$ & $\Delta$ & $\Delta$ & $\Delta$ & $\Delta$ & $\Delta$ & $\Delta$ & $\Delta$ & $\Delta$ & $\Delta$ & $\Delta$ \\
\hline 7 & $\Delta$ & $\Delta$ & $\Delta$ & $\Delta$ & $\Delta$ & $\triangle$ & $\triangle$ & $\triangle$ & $\triangle$ & $\Delta$ & $\Delta$ & $\triangle$ & $\triangle$ & $\triangle$ & $\triangle$ \\
\hline 6 & $\Delta$ & $\Delta$ & $\boldsymbol{\Delta}$ & $\Delta$ & $\Delta$ & $\Delta$ & $\Delta$ & $\Delta$ & $\Delta$ & $\Delta$ & $\Delta$ & $\Delta$ & $\Delta$ & $\Delta$ & $\Delta$ \\
\hline 5 & $\Delta$ & $\boldsymbol{\Delta}$ & $\boldsymbol{\Delta}$ & $\Delta$ & $\boldsymbol{\Delta}$ & $\Delta$ & $\Delta$ & $\Delta$ & $\Delta$ & $\Delta$ & $\Delta$ & $\Delta$ & $\Delta$ & $\Delta$ & $\Delta$ \\
\hline & \multicolumn{3}{|c|}{$R F_{0}=1.00$} & \multicolumn{3}{|c|}{$\mathrm{RF}_{1}=0.70$} & \multicolumn{3}{|c|}{$\mathrm{RF}_{2}=0.49$} & \multicolumn{3}{|c|}{$\mathrm{RF}_{3}=0.64$} & \multicolumn{3}{|c|}{$\mathrm{RF}_{4}=0.52$} \\
\hline
\end{tabular}

trained NN. On the other hand, (b) shows the BTD from FE and NN together with the accuracy of models from the literature. For instance, 'FSDT FE', indicates the accuracy of the first-order shear deformation theory as obtained via the FE model, whereas 'FSDT NN' refers to the output of the trained NN. Figures 8 and 9 report the results of a/h = 50 and 10, respectively, and Table 6 presents the numerical values related to the models from literature. The BTD models from NN are in Tables 7, 8 and 9. For instance, the six DOF best model for $\mathrm{a} / \mathrm{h}=10$ is the following:

$$
\begin{aligned}
& u_{x}=u_{x_{1}}+z u_{x_{2}}+z^{3} u_{x_{4}} \\
& u_{y}=u_{y_{1}}+z u_{y_{2}} \\
& u_{z}=u_{z_{1}}
\end{aligned}
$$

The last row of each table reports the relevance factor of the expansion orders (RF). The $R F$ is the ratio between the number of active instances and the total number of cases. For instance, $\mathrm{RF}_{0}=1$ indicates that the zeroth-order terms are always present in the BTD. The combined information stemming from the previous figures and tables is in Figure 10 for $\mathrm{a} / \mathrm{h}=10$ with the explicit indication of the seven, six, and five DOF best displacement fields. The results suggest that

- The proposed NN framework can detect the FE results with satisfactory accuracy. Two capabilities are relevant, namely, the possibility of using the $\mathrm{NN}$ to evaluate 
Table 15 Influence of a/h and lamination on some particular cases from Figs. 17 and 18

\begin{tabular}{llllll}
\hline & $\mathbf{a} / \mathbf{h}$ & Lamination & FSDT & PTD & TSDT \\
\hline $\mathrm{FE}$ & 75 & $0 / 90 / 0$ & 0.642 & 0.150 & 0.492 \\
$\mathrm{NN}$ & 75 & $0 / 90 / 0$ & 0.575 & 0.101 & 0.441 \\
$\mathrm{FE}$ & 25 & $0 / 90 / 0$ & 3.533 & 2.630 & 0.914 \\
$\mathrm{NN}$ & 25 & $0 / 90 / 0$ & 10.93 & 9.232 & 1.233 \\
$\mathrm{FE}$ & 75 & $0 / 90 / 0 / 90$ & 0.523 & 0.143 & 0.457 \\
$\mathrm{NN}$ & 75 & $0 / 90 / 0 / 90$ & 0.520 & 0.135 & 0.468 \\
$\mathrm{FE}$ & 25 & $0 / 90 / 0 / 90$ & 2.821 & 1.823 & 1.518 \\
$\mathrm{NN}$ & 25 & $0 / 90 / 0 / 90$ & 3.872 & 2.688 & 2.072 \\
\hline
\end{tabular}

Table 16 BTD models, 0/90/0, $\mathrm{a} / \mathrm{h}=75$

\begin{tabular}{|c|c|c|c|c|c|c|c|c|c|c|c|c|c|c|c|}
\hline DOF & $\mathbf{u}_{\mathbf{x} 1}$ & $\mathbf{u}_{\mathbf{y} 1}$ & $\mathbf{u}_{\mathrm{z} 1}$ & $u_{x 2}$ & $u_{y_{2}}$ & $\mathbf{u}_{\mathrm{z} 2}$ & $u_{\mathbf{x} 3}$ & $u_{y 3}$ & $\mathbf{u}_{\mathrm{z} 3}$ & $u_{\mathbf{x} 4}$ & $\mathbf{u}_{\mathbf{y} 4}$ & $\mathbf{u}_{\mathrm{z} 4}$ & $u_{\mathbf{x} 5}$ & $u_{y 5}$ & $\mathbf{u}_{\mathrm{z} 5}$ \\
\hline 15 & $\Delta$ & $\Delta$ & $\Delta$ & $\Delta$ & $\boldsymbol{\Delta}$ & $\Delta$ & $\Delta$ & $\Delta$ & $\Delta$ & $\Delta$ & $\Delta$ & $\Delta$ & $\boldsymbol{\Delta}$ & $\Delta$ & $\Delta$ \\
\hline 14 & $\Delta$ & $\boldsymbol{\Delta}$ & $\boldsymbol{\Delta}$ & $\Delta$ & $\Delta$ & $\Delta$ & $\boldsymbol{\Delta}$ & $\Delta$ & $\boldsymbol{\Delta}$ & $\Delta$ & $\triangle$ & $\boldsymbol{\Delta}$ & $\Delta$ & $\boldsymbol{\Delta}$ & $\boldsymbol{\Delta}$ \\
\hline 13 & $\Delta$ & $\Delta$ & $\Delta$ & $\Delta$ & $\Delta$ & $\Delta$ & $\Delta$ & $\triangle$ & $\Delta$ & $\Delta$ & $\Delta$ & $\Delta$ & $\Delta$ & $\Delta$ & $\Delta$ \\
\hline 12 & $\Delta$ & $\Delta$ & $\Delta$ & $\Delta$ & $\Delta$ & $\Delta$ & $\Delta$ & $\triangle$ & $\Delta$ & $\Delta$ & $\triangle$ & $\Delta$ & $\Delta$ & $\Delta$ & $\Delta$ \\
\hline 11 & $\Delta$ & $\Delta$ & $\boldsymbol{\Delta}$ & $\Delta$ & $\boldsymbol{\Delta}$ & $\Delta$ & $\triangle$ & $\triangle$ & $\Delta$ & $\Delta$ & $\triangle$ & $\Delta$ & $\triangle$ & $\Delta$ & $\boldsymbol{\Delta}$ \\
\hline 10 & $\Delta$ & $\Delta$ & $\Delta$ & $\Delta$ & $\Delta$ & $\Delta$ & $\triangle$ & $\triangle$ & $\Delta$ & $\Delta$ & $\triangle$ & $\Delta$ & $\triangle$ & $\triangle$ & $\Delta$ \\
\hline 9 & $\Delta$ & $\Delta$ & $\Delta$ & $\Delta$ & $\Delta$ & $\Delta$ & $\Delta$ & $\triangle$ & $\Delta$ & $\Delta$ & $\Delta$ & $\Delta$ & $\triangle$ & $\triangle$ & $\Delta$ \\
\hline 8 & $\Delta$ & $\Delta$ & $\Delta$ & $\Delta$ & $\Delta$ & $\Delta$ & $\triangle$ & $\triangle$ & $\Delta$ & $\Delta$ & $\triangle$ & $\triangle$ & $\triangle$ & $\triangle$ & $\triangle$ \\
\hline 7 & $\Delta$ & $\Delta$ & $\Delta$ & $\Delta$ & $\Delta$ & $\Delta$ & $\Delta$ & $\Delta$ & $\Delta$ & $\Delta$ & $\Delta$ & $\Delta$ & $\triangle$ & $\Delta$ & $\Delta$ \\
\hline 6 & $\Delta$ & $\Delta$ & $\Delta$ & $\Delta$ & $\Delta$ & $\triangle$ & $\triangle$ & $\triangle$ & $\Delta$ & $\triangle$ & $\triangle$ & $\triangle$ & $\triangle$ & $\triangle$ & $\triangle$ \\
\hline 5 & $\Delta$ & $\Delta$ & $\Delta$ & $\Delta$ & $\Delta$ & $\triangle$ & $\Delta$ & $\Delta$ & $\Delta$ & $\Delta$ & $\Delta$ & $\Delta$ & $\triangle$ & $\Delta$ & $\Delta$ \\
\hline & \multicolumn{3}{|c|}{$\mathrm{RF}_{0}=1.00$} & \multicolumn{3}{|c|}{$\mathrm{RF}_{1}=0.91$} & \multicolumn{3}{|c|}{$\mathrm{RF}_{2}=0.46$} & \multicolumn{3}{|c|}{$\mathrm{RF}_{3}=0.49$} & \multicolumn{3}{|c|}{$\mathrm{RF}_{4}=0.49$} \\
\hline
\end{tabular}

theories from the literature and the ability to cover the discontinuous error range entirely.

- The discontinuity in the error range, i.e., the presence of accuracy bands indicates that there may not exist structural theories satisfying a given error requirement. As shown in [76], such gaps widen as the thickness ratio increases. For thin shells, the lower-order terms, i.e., the FSDT variables, play a decisive role, and their absence causes high errors. As the shell is thicker, higher-order terms gain relevance leading to more homogeneous error distributions.

- There are no relevant differences in the BTD for $\mathrm{a} / \mathrm{h}=100$ and 50 except that the latter has a broader error range as the five DOF model, coinciding with the FSDT, yields a $2 \%$ error. The models from the literature, although not always on the BTD curve, provide satisfactory accuracies.

- For $\mathrm{a} / \mathrm{h}=10$, at least six DOF are necessary to have errors smaller than $1 \%$ and the variables required to meet such a requirement are the cubic in-plane ones.

- The analysis of the RF shows that, as well-known, for thin shells, zeroth- and firstorder variables are the most relevant. As the thickness increases, the third-order terms gain importance with smaller relevance for first-order ones. The NN detected very similar RF as compared to FE from [76], meaning that the prosed framework can detect the accuracy of a given structural model and determine the models on the BTD curve reliably.

Further analyses concerned the comparison of NN with linear regression (LR). LR is computationally cheaper than $\mathrm{NN}$ and can provide explicit weights related to each training 
Table 17 BTD models, 0/90/0, a/h $=25$

\begin{tabular}{|c|c|c|c|c|c|c|c|c|c|c|c|c|c|c|c|}
\hline DOF & $\mathbf{u}_{\mathbf{x} 1}$ & $\mathbf{u}_{\mathbf{y} \mathbf{1}}$ & $\mathbf{u}_{\mathrm{z} \mathbf{1}}$ & $u_{x 2}$ & $u_{y 2}$ & $\mathbf{u}_{\mathrm{z} 2}$ & $\mathbf{u}_{\mathbf{x} 3}$ & $\mathbf{u}_{\mathrm{y} 3}$ & $\mathbf{u}_{\mathrm{z} 3}$ & $\mathbf{u}_{\mathbf{x} 4}$ & $u_{y 4}$ & $\mathbf{u}_{\mathbf{z} 4}$ & $u_{x 5}$ & $u_{y 5}$ & $\mathbf{u}_{\mathrm{z} 5}$ \\
\hline 15 & $\boldsymbol{\Delta}$ & $\boldsymbol{\Delta}$ & $\boldsymbol{\Delta}$ & $\Delta$ & $\boldsymbol{\Delta}$ & $\boldsymbol{\Delta}$ & $\Delta$ & $\Delta$ & $\Delta$ & $\Delta$ & $\Delta$ & $\boldsymbol{\Delta}$ & $\Delta$ & $\boldsymbol{\Delta}$ & $\Delta$ \\
\hline 14 & $\Delta$ & $\Delta$ & $\Delta$ & $\Delta$ & $\Delta$ & $\Delta$ & $\Delta$ & $\Delta$ & $\Delta$ & $\Delta$ & $\Delta$ & $\Delta$ & $\Delta$ & $\Delta$ & $\triangle$ \\
\hline 13 & $\Delta$ & $\Delta$ & $\Delta$ & $\Delta$ & $\Delta$ & $\Delta$ & $\Delta$ & $\Delta$ & $\Delta$ & $\Delta$ & $\Delta$ & $\Delta$ & $\Delta$ & $\Delta$ & $\triangle$ \\
\hline 12 & $\Delta$ & $\Delta$ & $\Delta$ & $\Delta$ & $\Delta$ & $\Delta$ & $\Delta$ & $\Delta$ & $\Delta$ & $\Delta$ & $\Delta$ & $\Delta$ & $\Delta$ & $\Delta$ & $\Delta$ \\
\hline 11 & $\Delta$ & $\Delta$ & $\Delta$ & $\Delta$ & $\Delta$ & $\Delta$ & $\Delta$ & $\triangle$ & $\Delta$ & $\Delta$ & $\Delta$ & $\Delta$ & $\triangle$ & $\triangle$ & $\triangle$ \\
\hline 10 & $\Delta$ & $\Delta$ & $\Delta$ & $\Delta$ & $\Delta$ & $\Delta$ & $\triangle$ & $\Delta$ & $\Delta$ & $\Delta$ & $\Delta$ & $\Delta$ & $\triangle$ & $\Delta$ & $\Delta$ \\
\hline 9 & $\Delta$ & $\Delta$ & $\Delta$ & $\Delta$ & $\Delta$ & $\Delta$ & $\triangle$ & $\triangle$ & $\Delta$ & $\Delta$ & $\Delta$ & $\triangle$ & $\triangle$ & $\triangle$ & $\triangle$ \\
\hline 8 & $\boldsymbol{\Delta}$ & $\Delta$ & $\Delta$ & $\Delta$ & $\Delta$ & $\Delta$ & $\triangle$ & $\triangle$ & $\Delta$ & $\Delta$ & $\Delta$ & $\Delta$ & $\triangle$ & $\triangle$ & $\triangle$ \\
\hline 7 & $\Delta$ & $\Delta$ & $\Delta$ & $\Delta$ & $\Delta$ & $\Delta$ & $\Delta$ & $\Delta$ & $\Delta$ & $\Delta$ & $\Delta$ & $\Delta$ & $\Delta$ & $\Delta$ & $\Delta$ \\
\hline 6 & $\Delta$ & $\Delta$ & $\Delta$ & $\Delta$ & $\Delta$ & $\triangle$ & $\triangle$ & $\triangle$ & $\triangle$ & $\Delta$ & $\Delta$ & $\triangle$ & $\triangle$ & $\triangle$ & $\triangle$ \\
\hline 5 & $\Delta$ & $\boldsymbol{\Delta}$ & $\Delta$ & $\Delta$ & $\Delta$ & $\Delta$ & $\Delta$ & $\Delta$ & $\Delta$ & $\Delta$ & $\Delta$ & $\Delta$ & $\Delta$ & $\Delta$ & $\Delta$ \\
\hline & \multicolumn{3}{|c|}{$R F_{0}=1.00$} & \multicolumn{3}{|c|}{$\mathrm{RF}_{1}=0.91$} & \multicolumn{3}{|c|}{$\mathrm{RF}_{2}=0.49$} & \multicolumn{3}{|c|}{$\mathrm{RF}_{3}=0.70$} & \multicolumn{3}{|c|}{$\mathrm{RF}_{4}=0.24$} \\
\hline
\end{tabular}

feature. Figures 11 and 12 show the results for two training sets; namely, 10 and 100\%. The accuracy of LR is acceptable just in the second case but lower than NN.

0/90/0/90

The second numerical case investigated the effect of an asymmetric lamination on the BTD. All other parameters remained as those of the previous case. Table 10 presents the transverse displacement values with comparisons with other models from literature, when available. Figures 13, 14 and 15 show the BTD from FE and NN, and Table 11 presents the numerical values of the models from literature. For $\mathrm{a} / \mathrm{h}=50$ and 10 , the NN had three layers of ten neurons as one layer was not enough to fit the BTD curve that, in these cases, presents a more irregular shape than for $\mathrm{a} / \mathrm{h}=100$. Tables 12,13 and 14 show the BTD models and relevance factors. Figure 16 shows the BTD curve for $\mathrm{a} / \mathrm{h}=10$ and the displacement field retrieved from Table 14. The results show that

- As mentioned, a more complex NN architecture was necessary, and the match between FE and NN BTD is not perfect. Some differences are visible for higher DOF models. However, such differences are still acceptable, given that, in the worst case, remain within the $1 \%$ error range. The BTD curve presents various portions having different shapes leading to a more difficult curve fitting.

- As in the previous case, $\mathrm{a} / \mathrm{h}=100$ and 50 have similar BTD, and seven DOF are enough to have very low errors with the FSDT providing accurate results.

- For $\mathrm{a} / \mathrm{h}=10,11$ DOF are necessary to have an error lower than $1 \%$ with full fourthorder expansions for the in-plane terms. Besides linear terms, the third-order terms are decisive for their absence leading to errors around $10 \%$.

- The considered models from the literature provide high accuracy for thin shells. On the other hand, for $\mathrm{a} / \mathrm{h}=10$, only the TSDT can provide acceptable accuracy.

$a / h$ as a training variable

This section concerns the use of the thickness ratio as an additional training variable. The aim is to show the possibility of using NN to test structural theories and obtain results as the typical parameters of the structure change without the need of creating and running a new FE analysis. In this section, the training inputs are 13, i.e., 12 generalized displacement 


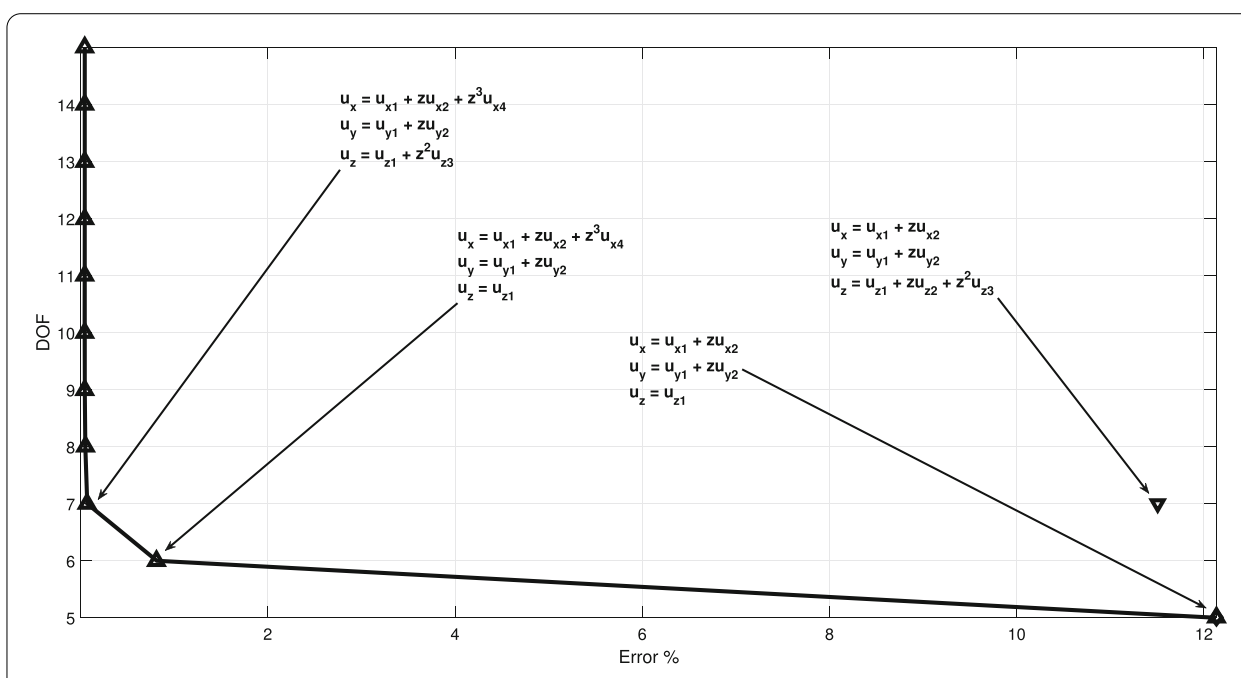

Fig. 10 BTD for 0/90/0, a/h = 10 with seven, six and five DOF models indicated

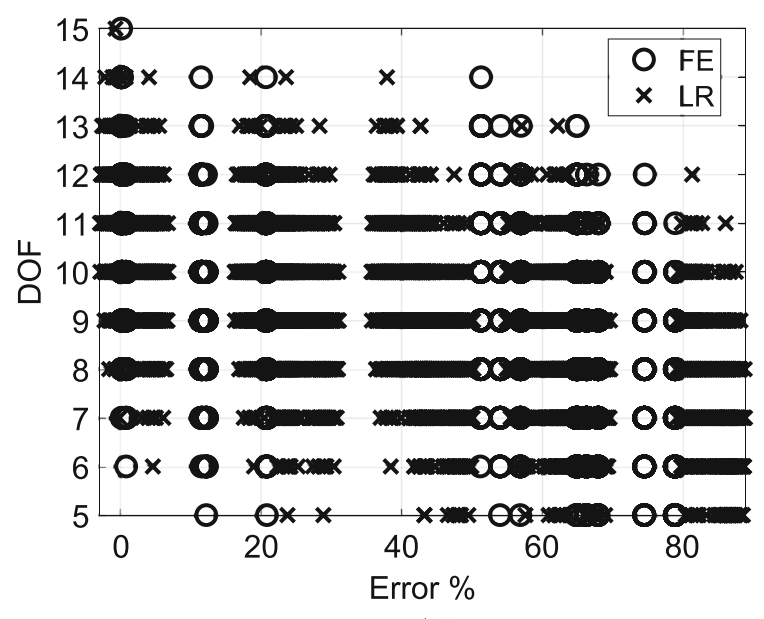

a All

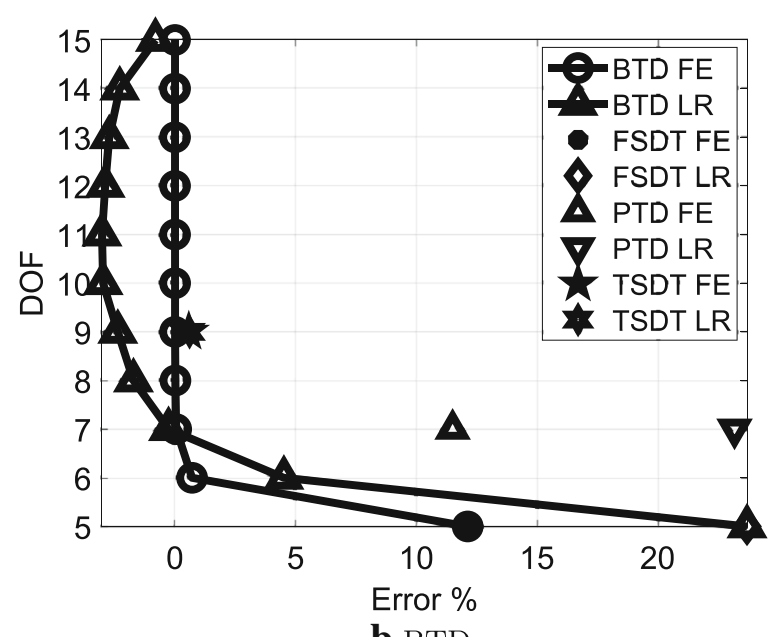

Fig. $11 \mathrm{O} / 90 / 0, \mathrm{a} / \mathrm{h}=10$, comparison between $\mathrm{FE}$ and linear regression (LR), 10\% training set 
Table 18 BTD models, 0/90/0/90, $\mathrm{a} / \mathrm{h}=75$

\begin{tabular}{|c|c|c|c|c|c|c|c|c|c|c|c|c|c|c|c|}
\hline DOF & $u_{\mathbf{x} 1}$ & $u_{\mathbf{y} 1}$ & $\mathbf{u}_{\mathrm{z} 1}$ & $u_{x 2}$ & $\mathrm{u}_{\mathrm{y} 2}$ & $\mathbf{u}_{\mathrm{z} 2}$ & $\mathbf{u}_{\mathbf{x} 3}$ & $\mathrm{u}_{\mathrm{y} 3}$ & $\mathbf{u}_{\mathrm{z} 3}$ & $u_{x 4}$ & $\mathrm{u}_{\mathrm{y} 4}$ & $\mathbf{u}_{\mathrm{z} 4}$ & $u_{x 5}$ & $u_{y 5}$ & $\mathbf{u}_{\mathrm{z} 5}$ \\
\hline 15 & $\Delta$ & $\Delta$ & $\Delta$ & $\Delta$ & $\Delta$ & $\Delta$ & $\Delta$ & $\Delta$ & $\Delta$ & $\Delta$ & $\Delta$ & $\Delta$ & $\Delta$ & $\Delta$ & $\Delta$ \\
\hline 14 & $\Delta$ & $\Delta$ & $\Delta$ & $\Delta$ & $\Delta$ & $\Delta$ & $\Delta$ & $\Delta$ & $\Delta$ & $\Delta$ & $\Delta$ & $\Delta$ & $\Delta$ & $\Delta$ & $\Delta$ \\
\hline 13 & $\Delta$ & $\Delta$ & $\Delta$ & $\Delta$ & $\Delta$ & $\Delta$ & $\Delta$ & $\Delta$ & $\Delta$ & $\Delta$ & $\Delta$ & $\Delta$ & $\Delta$ & $\Delta$ & $\Delta$ \\
\hline 12 & $\Delta$ & $\Delta$ & $\Delta$ & $\Delta$ & $\Delta$ & $\Delta$ & $\Delta$ & $\Delta$ & $\Delta$ & $\Delta$ & $\Delta$ & $\Delta$ & $\Delta$ & $\Delta$ & $\Delta$ \\
\hline 11 & $\Delta$ & $\Delta$ & $\Delta$ & $\Delta$ & $\Delta$ & $\Delta$ & $\Delta$ & $\Delta$ & $\Delta$ & $\Delta$ & $\Delta$ & $\Delta$ & $\Delta$ & $\Delta$ & $\Delta$ \\
\hline 10 & $\Delta$ & $\Delta$ & $\Delta$ & $\Delta$ & $\Delta$ & $\Delta$ & $\Delta$ & $\Delta$ & $\Delta$ & $\Delta$ & $\Delta$ & $\Delta$ & $\Delta$ & $\Delta$ & $\Delta$ \\
\hline 9 & $\Delta$ & $\Delta$ & $\Delta$ & $\Delta$ & $\Delta$ & $\Delta$ & $\Delta$ & $\Delta$ & $\Delta$ & $\Delta$ & $\Delta$ & $\Delta$ & $\Delta$ & $\Delta$ & $\Delta$ \\
\hline 8 & $\Delta$ & $\Delta$ & $\Delta$ & $\Delta$ & $\Delta$ & $\Delta$ & $\Delta$ & $\Delta$ & $\Delta$ & $\Delta$ & $\Delta$ & $\Delta$ & $\Delta$ & $\Delta$ & $\Delta$ \\
\hline 7 & $\Delta$ & $\Delta$ & $\Delta$ & $\Delta$ & $\Delta$ & $\Delta$ & $\Delta$ & $\Delta$ & $\Delta$ & $\Delta$ & $\Delta$ & $\Delta$ & $\Delta$ & $\Delta$ & $\Delta$ \\
\hline 6 & $\Delta$ & $\Delta$ & $\Delta$ & $\Delta$ & $\Delta$ & $\Delta$ & $\Delta$ & $\Delta$ & $\Delta$ & $\Delta$ & $\Delta$ & $\Delta$ & $\Delta$ & $\Delta$ & $\Delta$ \\
\hline 5 & $\Delta$ & $\Delta$ & $\Delta$ & $\Delta$ & $\Delta$ & $\Delta$ & $\Delta$ & $\Delta$ & $\Delta$ & $\Delta$ & $\Delta$ & $\Delta$ & $\Delta$ & $\Delta$ & $\Delta$ \\
\hline & \multicolumn{3}{|c|}{$\mathrm{RF}_{0}=1.00$} & \multicolumn{3}{|c|}{$\mathrm{RF}_{1}=0.70$} & \multicolumn{3}{|c|}{$\mathrm{RF}_{2}=0.67$} & \multicolumn{3}{|c|}{$\mathrm{RF}_{3}=0.55$} & \multicolumn{3}{|c|}{$\mathrm{RF}_{4}=0.42$} \\
\hline
\end{tabular}

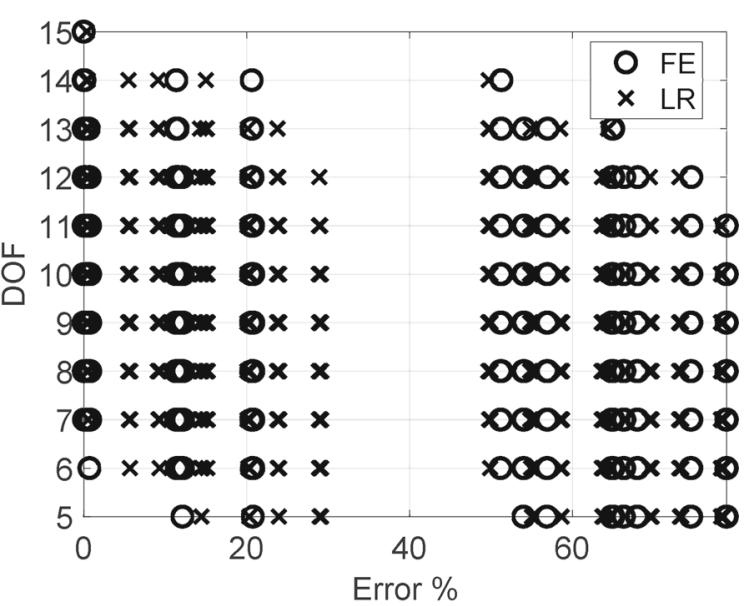

a All

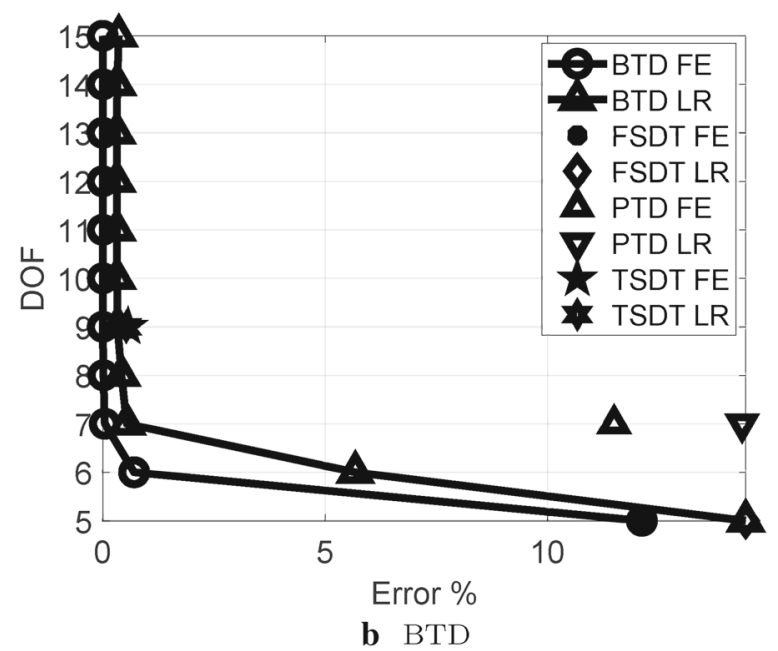

Fig. $12 \mathrm{O} / 90 / 0, \mathrm{a} / \mathrm{h}=10$, comparison between $\mathrm{FE}$ and linear regression (LR), 100\% training set 

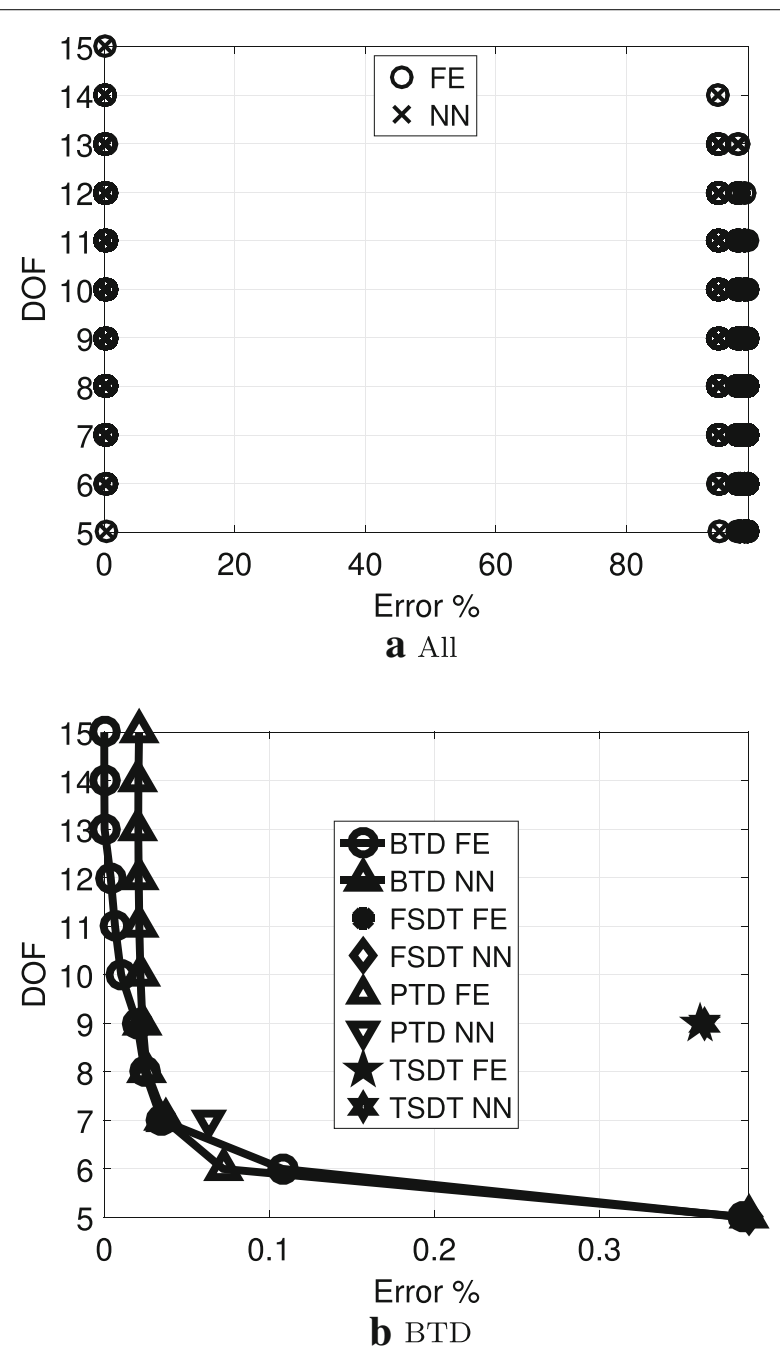

Fig. $13 \mathrm{FE}$ and $\mathrm{NN}$ results for $0 / 90 / 0 / 90, \mathrm{a} / \mathrm{h}=100$, one layer with ten neurons

variables and the thickness ratio. The $\mathrm{NN}$ had three layers with ten neurons each. The results refer to the two lamination schemes of the previous sections and $\mathrm{a} / \mathrm{h}=100,50$ and 10 were the training sets, i.e., the training set size was $10 \%$ of $3 \times 2^{12}$. a/h $=25$ and 75 are the thickness ratios evaluated via the trained NN. The BTD curves are in Figs. 17 and 18, values from selected models are in Table 15, whereas the BTD models in Tables 16, 17, 18, and 19. The results suggest that

- There is a good match between the FE and NN results. Some differences are observable for $\mathrm{a} / \mathrm{h}=25$ but within the $1 \%$ error range at worst.

- $\mathrm{a} / \mathrm{h}=25$ tends to have similar curves and BTD models of $\mathrm{a} / \mathrm{h}=10$ with the increasing relevance of third-order terms. Such a tendency may explain the better match of FE and $\mathrm{NN}$ for thin shells given that the training used $\mathrm{a} / \mathrm{h}=100,50$, and 10 . As seen in the previous sections, the latter case tends to have BTD curves quite different as compared to the first two cases. 


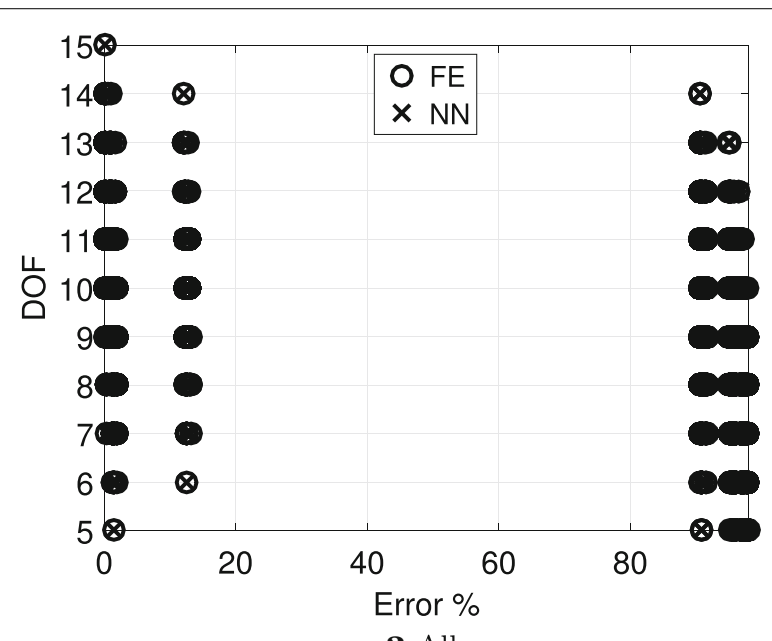

a All

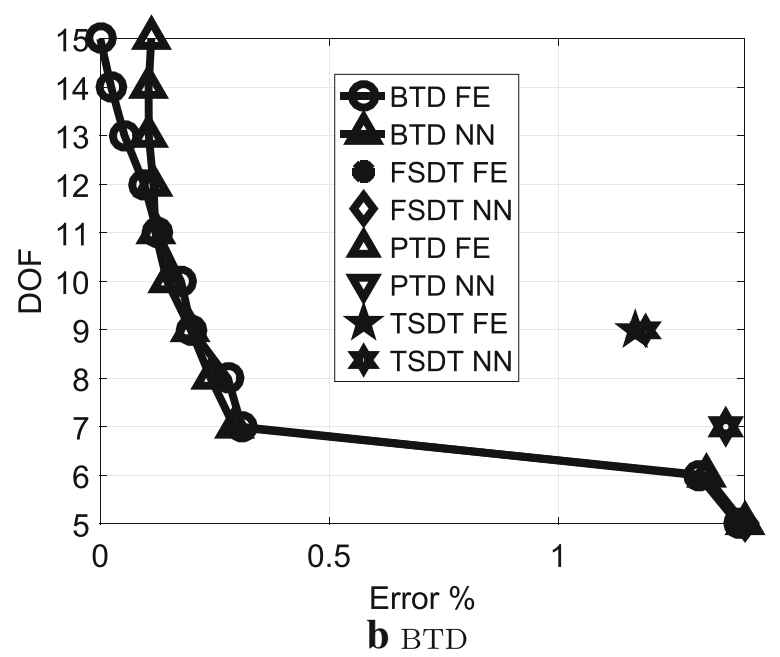

Fig. $14 \mathrm{FE}$ and $\mathrm{NN}$ results for $0 / 90 / 0 / 90, \mathrm{a} / \mathrm{h}=50$, three layers with ten neurons

- The trained NN provides outputs concerning models from the literature with good accuracy except for the PTD in the moderately thick, symmetric lamination case.

\section{Conclusions}

This paper presented a new approach to evaluating the accuracy of shell models for composites via the use of neural networks $(\mathrm{NN})$. The $\mathrm{NN}$ training used results from shell finite elements (FE) stemming from the Carrera Unified Formulation (CUF) and adopting a 15 DOF, fourth-order polynomial expansion along the thickness as the reference solution. The first set of training inputs considered one-tenth of the combinations of active and inactive terms, i.e., keeping the constant terms always active, one-tenth of $2^{12}$ shell theories. The second set of inputs added the thickness ratio as a further variable. In all cases, the target was the maximum transverse displacement of a square, simply-supported shell under bi-sinusoidal transverse load. The NN architecture ranged from one to three layers, with ten neurons each. The result verification exploited the FE results of all $2^{12}$ cases. The 


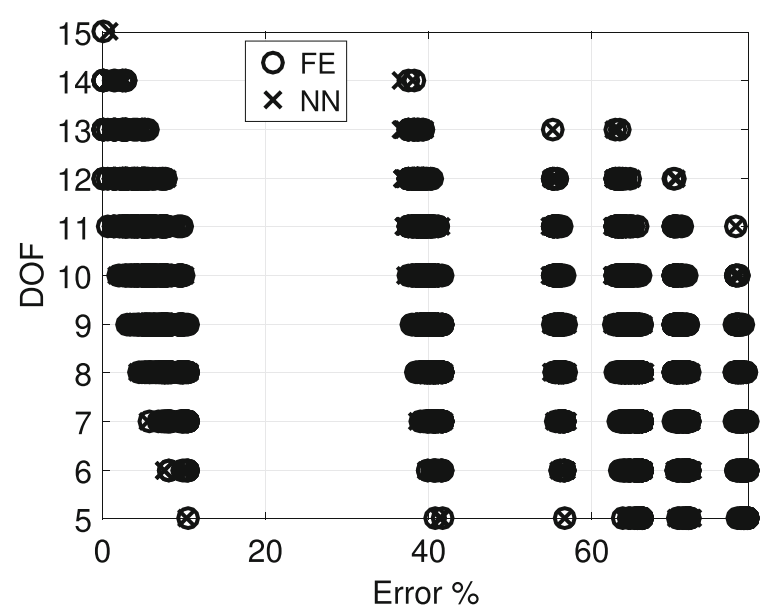

a All

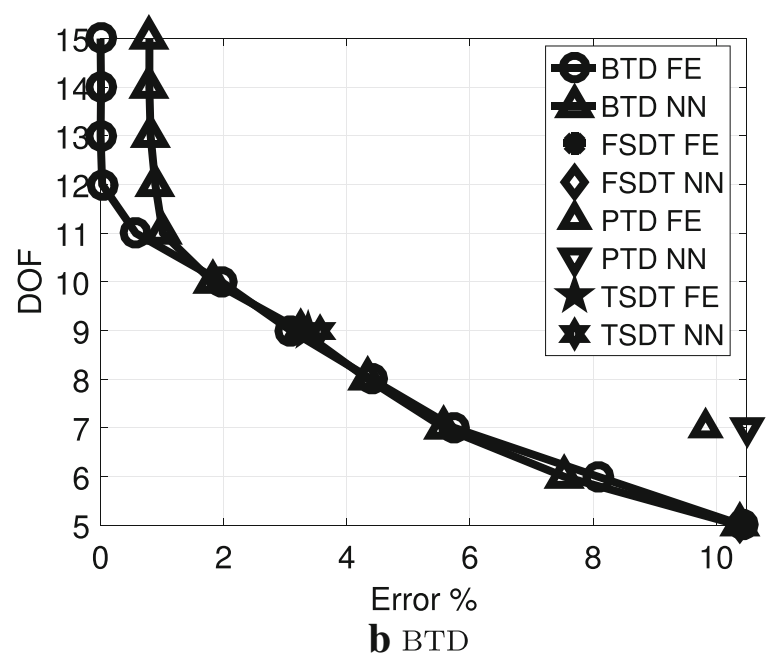

Fig. $15 \mathrm{FE}$ and NN results for 0/90/0/90, $\mathrm{a} / \mathrm{h}=10$, three layers with ten neurons

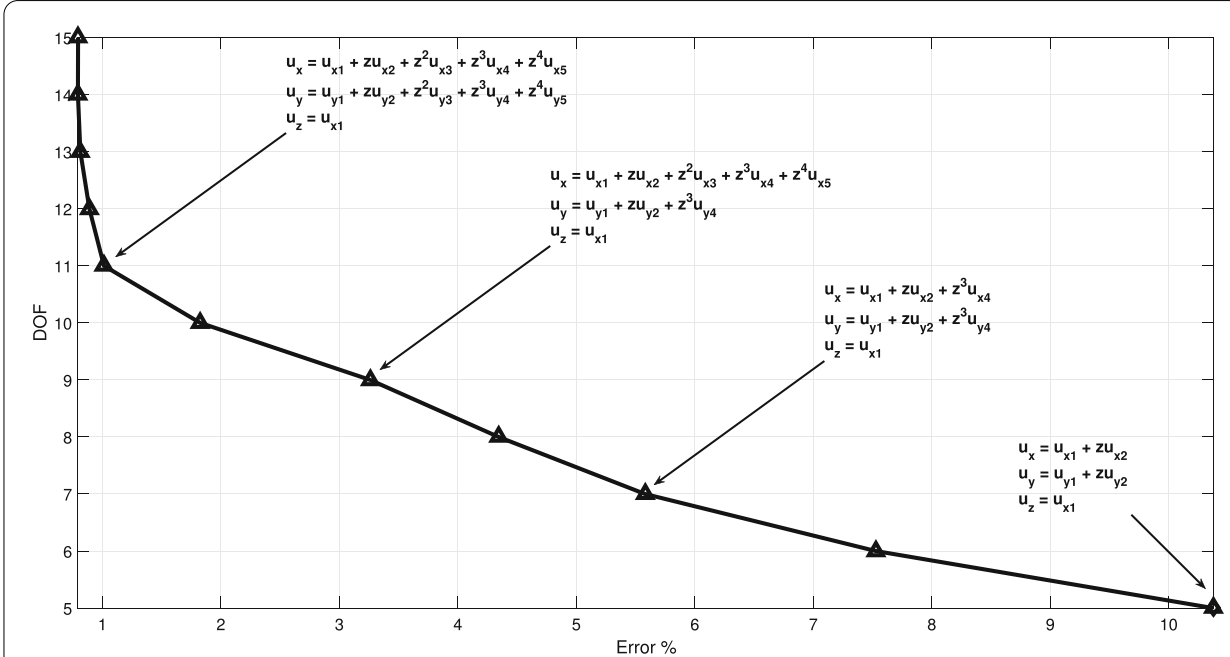

Fig. 16 BTD for 0/90/0/90, a/h = 10 with eleven, nine, seven and five DOF models indicated 


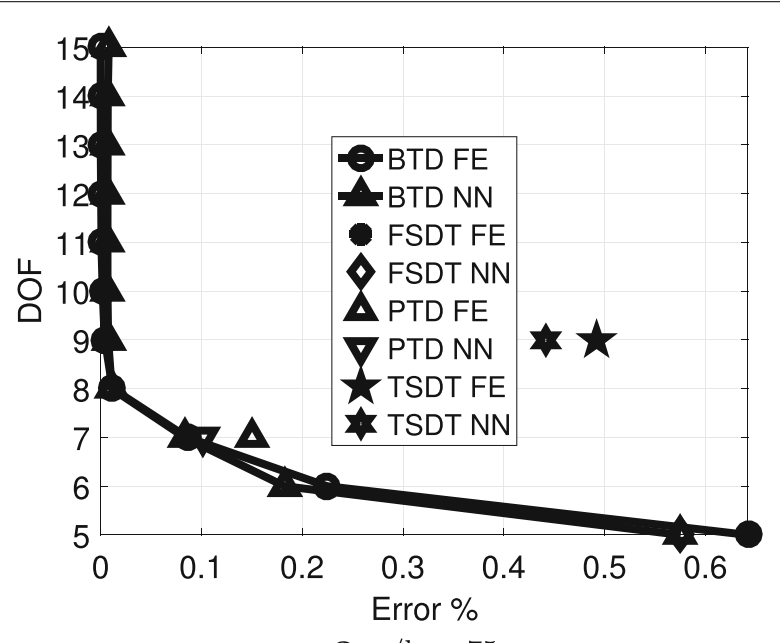

a $\mathrm{a} / \mathrm{h}=75$

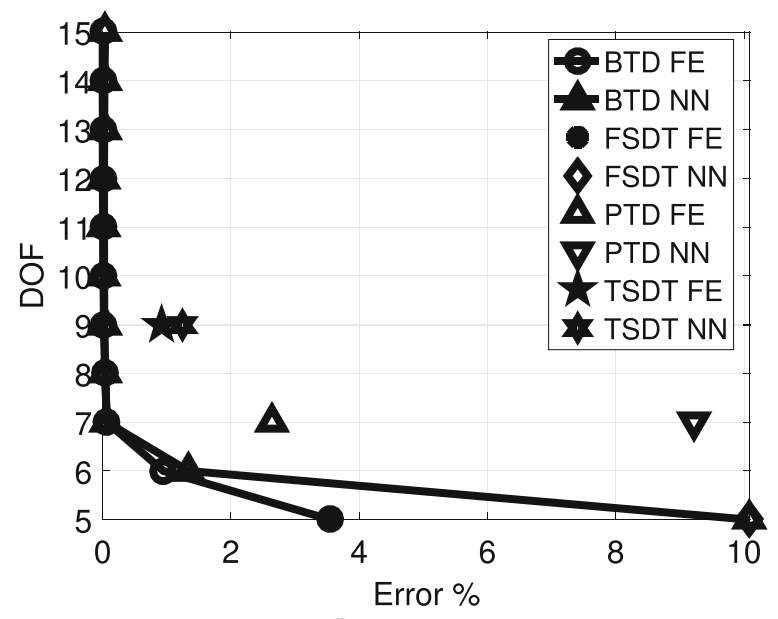

b $\mathrm{a} / \mathrm{h}=25$

Fig. $17 \mathrm{FE}$ and $\mathrm{NN}$ results for $0 / 90 / 0, \mathrm{a} / \mathrm{h}=25$ and 75 , three layers with ten neurons

Table 19 BTD models, 0/90/0/90, a/h $=25$

\begin{tabular}{|c|c|c|c|c|c|c|c|c|c|c|c|c|c|c|c|}
\hline DOF & $u_{x 1}$ & $\mathrm{u}_{\mathrm{y} 1}$ & $\mathbf{u}_{\mathrm{z} 1}$ & $u_{x 2}$ & $\mathrm{u}_{\mathrm{y} 2}$ & $\mathbf{u}_{\mathrm{z} 2}$ & $\mathbf{u}_{\mathbf{x} 3}$ & $\mathrm{u}_{\mathrm{y} 3}$ & $\mathbf{u}_{\mathrm{z} 3}$ & $\mathrm{u}_{\mathrm{x} 4}$ & $\mathrm{u}_{\mathrm{y} 4}$ & $\mathrm{u}_{\mathrm{z} 4}$ & $u_{x 5}$ & $\mathrm{u}_{\mathrm{y} 5}$ & $\mathbf{u}_{\mathrm{z} 5}$ \\
\hline 15 & $\Delta$ & $\Delta$ & $\Delta$ & $\Delta$ & $\Delta$ & $\Delta$ & $\Delta$ & $\Delta$ & $\Delta$ & $\Delta$ & $\Delta$ & $\Delta$ & $\Delta$ & $\Delta$ & $\Delta$ \\
\hline 14 & $\Delta$ & $\Delta$ & $\Delta$ & $\Delta$ & $\Delta$ & $\Delta$ & $\Delta$ & $\Delta$ & $\Delta$ & $\Delta$ & $\Delta$ & $\Delta$ & $\Delta$ & $\Delta$ & $\Delta$ \\
\hline 13 & $\Delta$ & $\Delta$ & $\Delta$ & $\Delta$ & $\Delta$ & $\Delta$ & $\Delta$ & $\Delta$ & $\Delta$ & $\Delta$ & $\Delta$ & $\Delta$ & $\Delta$ & $\Delta$ & $\Delta$ \\
\hline 12 & $\Delta$ & $\Delta$ & $\Delta$ & $\Delta$ & $\Delta$ & $\Delta$ & $\Delta$ & $\Delta$ & $\Delta$ & $\Delta$ & $\Delta$ & $\Delta$ & $\Delta$ & $\Delta$ & $\Delta$ \\
\hline 11 & $\Delta$ & $\Delta$ & $\Delta$ & $\Delta$ & $\Delta$ & $\Delta$ & $\Delta$ & $\Delta$ & $\Delta$ & $\Delta$ & $\Delta$ & $\Delta$ & $\Delta$ & $\Delta$ & $\Delta$ \\
\hline 10 & $\Delta$ & $\Delta$ & $\Delta$ & $\Delta$ & $\Delta$ & $\Delta$ & $\Delta$ & $\Delta$ & $\Delta$ & $\Delta$ & $\Delta$ & $\Delta$ & $\Delta$ & $\Delta$ & $\Delta$ \\
\hline 9 & $\Delta$ & $\Delta$ & $\Delta$ & $\Delta$ & $\Delta$ & $\Delta$ & $\Delta$ & $\Delta$ & $\Delta$ & $\Delta$ & $\Delta$ & $\Delta$ & $\Delta$ & $\Delta$ & $\Delta$ \\
\hline 8 & $\Delta$ & $\Delta$ & $\Delta$ & $\Delta$ & $\Delta$ & $\Delta$ & $\Delta$ & $\Delta$ & $\Delta$ & $\Delta$ & $\Delta$ & $\Delta$ & $\Delta$ & $\Delta$ & $\Delta$ \\
\hline 7 & $\Delta$ & $\Delta$ & $\Delta$ & $\Delta$ & $\Delta$ & $\Delta$ & $\Delta$ & $\Delta$ & $\Delta$ & $\Delta$ & $\Delta$ & $\Delta$ & $\Delta$ & $\Delta$ & $\Delta$ \\
\hline 6 & $\Delta$ & $\Delta$ & $\Delta$ & $\Delta$ & $\Delta$ & $\Delta$ & $\Delta$ & $\Delta$ & $\Delta$ & $\Delta$ & $\Delta$ & $\Delta$ & $\Delta$ & $\Delta$ & $\Delta$ \\
\hline 5 & $\Delta$ & $\Delta$ & $\Delta$ & $\Delta$ & $\Delta$ & $\Delta$ & $\Delta$ & $\Delta$ & $\Delta$ & $\Delta$ & $\Delta$ & $\Delta$ & $\Delta$ & $\Delta$ & $\Delta$ \\
\hline & \multicolumn{3}{|c|}{$\mathrm{RF}_{0}=1.00$} & \multicolumn{3}{|c|}{$\mathrm{RF}_{1}=0.70$} & \multicolumn{3}{|c|}{$\mathrm{RF}_{2}=0.67$} & \multicolumn{3}{|c|}{$\mathrm{RF}_{3}=0.58$} & \multicolumn{3}{|c|}{$\mathrm{RF}_{4}=0.40$} \\
\hline
\end{tabular}




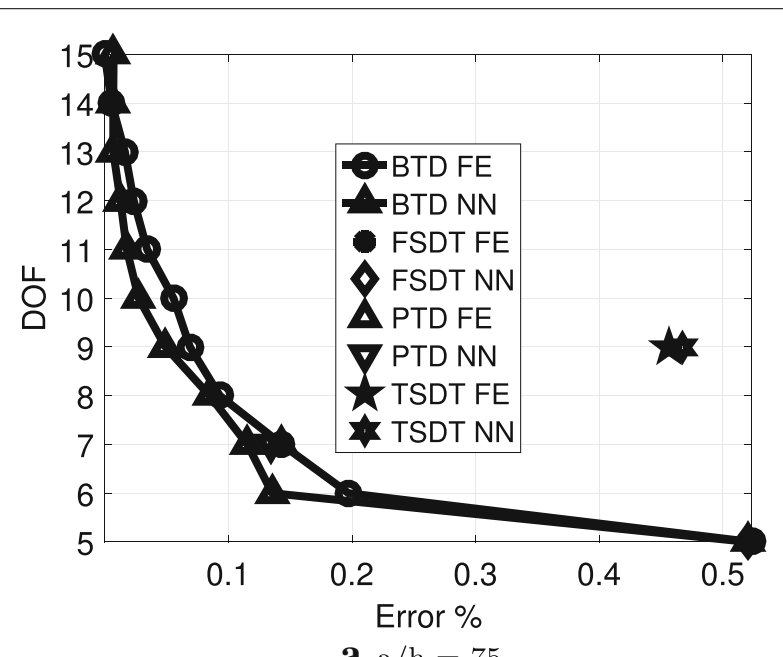

a $\mathrm{a} / \mathrm{h}=75$

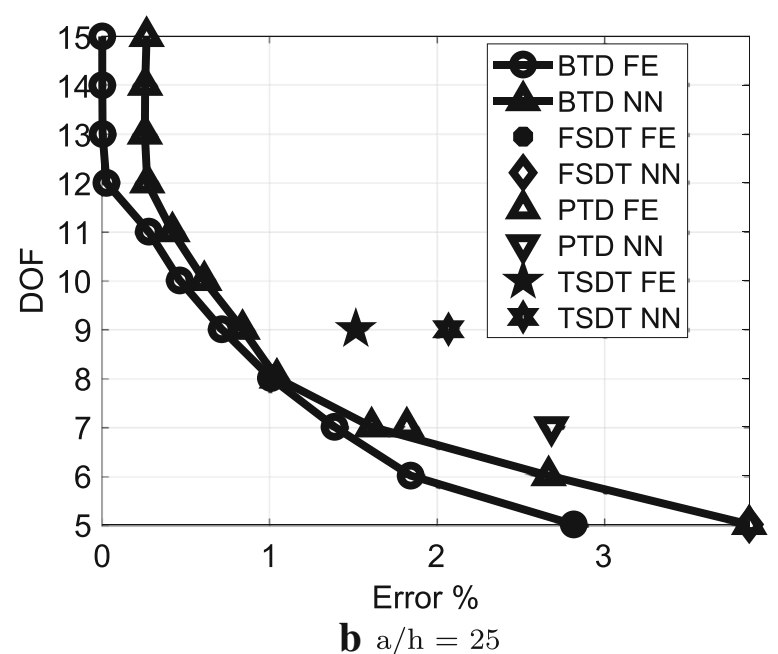

Fig. 18 FE and NN results for 0/90/0/90, $\mathrm{a} / \mathrm{h}=25$ and 75, three layers with ten neurons

NN provided the Best Theory Diagram (BTD), i.e., a curve giving the computationally cheapest model for a given accuracy. The BTD permits to evaluate the accuracy of any structural model and provides guidelines on the relevance of each generalized displacement variable. The main findings of this paper are the following:

- The use of NN proved to be valid as matched very well the FE solutions. The main convenience of $\mathrm{NN}$ is in the use of some $10 \%$ of $\mathrm{FE}$ analyses for training to obtain the BTD and evaluate the accuracy of a structural model without the need for further FE analyses.

- The NN training can incorporate physical features of the problems such as the thickness ratio allowing to obtain results without the need of new FE analyses and preprocessing.

- Potential critical aspects of this approach emerged as the training considered simultaneously thin and thick shells. Such a scenario required the use of more hidden layers and needs further investigations. 
- The BTD stemming from the NN matched very well those obtained via FE and presented in [76]. As well-known, the third-order in-plane terms are the most relevant variables to include to refine classical theories.

- Most of the models from literature provides good accuracy, although increments in thickness or asymmetric laminations can make such models inaccurate.

The combined use of CUF and NN is promising given that the former can provide thousands of data sets in minutes and benchmarking for the rigorous assessment of results, the latter can boost the computational efficiency and widen the applicability of virtual modeling. Future investigations should focus on the use of NN for multiple targets, e.g., multi-point stress values, and inverse problems to establish the best model by inputting the accuracy requirement.

\section{Acknowledgements}

Not applicable.

\section{Authors' contributions}

Professor Carrera and Dr. Petrolo equally contributed to the development of the CUF-AAM-NN framework. All authors read and approved the final manuscript.

\section{Funding}

Not applicable.

\section{Availability of data and materials}

Data are available upon request.

\section{Competing interests}

The authors declare that they have no competing interests.

Received: 30 July 2019 Accepted: 11 June 2020

Published online: 07 July 2020

\section{References}

1. Kirchhoff G. Uber das gleichgewicht und die bewegung einer elastischen scheibe. J fur reins und angewandte Mathematik. 1850;40:51-88.

2. Reissner E. The effect of transverse shear deformation on the bending of elastic plates. J Appl Mech. 1945;12:69-76.

3. Mindlin RD. Influence of rotatory inertia and shear in flexural motions of isotropic elastic plates. J Appl Mech. 1951;18:1031-6.

4. Carrera E. Developments, ideas and evaluations based upon the Reissner's mixed variational theorem in the modeling of multilayered plates and shells. Appl Mech Rev. 2001;54:301-29.

5. Washizu K. Variational methods in elasticity and plasticity. Oxford: Pergamon; 1968.

6. Kapania K, Raciti S. Recent advances in analysis of laminated beams and plates, part I: shear effects and buckling. AIAA J. 1989;27(7):923-35.

7. MacNeal RH. Perspective on finite elements for shell analysis. Finite Elem Anal Des. 1998;30(3):175-86.

8. Krätzig WB, Jun D. On 'best' shell models_from classical shells, degenerated and multi-layered concepts to 3D. Arch Appl Mech. 2003;73(1):1-25

9. Reddy JN, Arcinega RA. Shear deformation plate and shell theories: from Stavsky to present. Mech Adv Mater Struct. 2004;11(6):535-82.

10. Reddy JN. Exact solutions of moderately thick laminated shells. J Eng Mech. 1984:110(5):794-809.

11. Ren JG. Exact solutions for laminated cylindrical shells in cylindrical bending. Compos Sci Technol. 1987;29(3):169-87.

12. Leissa AW, Chang JD. Elastic deformation of thick, laminated composite shells. Compos Struct. 1996;35(2):153-70.

13. Shu XP. A refined theory of laminated shells with higher-order transverse shear deformation. Int J Solids Struct. 1997:34(6):673-83.

14. Wang X, Wang C, Yu ZY. An analytic method for interlaminar stress in a laminated cylindrical shell. Mech Adv Mater Struct. 2002;9(2):119-31.

15. Oktem AS, Chaudhuri RA. Fourier analysis of thick cross-ply Levy type clamped doubly-curved panels. Compos Struct. 2007;80(4):489-503.

16. Wu CP, Liu CC. Stress and displacement of thick doubly curved laminated shells. J Eng Mech. 1994;120(7):1403-28.

17. Noor AK, Rarig PL. Three-dimensional solutions of laminated cylinders. Comput Methods Appl Mech Eng. 1974:3(3):319-34.

18. Varadan TK, Bhaskar K. Bending of laminated orthotropic cylindrical shells—an elasticity approach. Compos Struct. 1991:17(2):141-56

19. Fan J, Zhang J. Analytical solutions for thick, doubly curved, laminated shells. J Eng Mech. 1992;118(7):1338-56. 
20. Bhimaraddi A, Chandrashekhara K. Three-dimensional elasticity solution for static response of simply supported orthotropic cylindrical shells. Compos Struct. 1992;20(4):227-35.

21. Wu CP, Tarn JQ, Chi SM. Three-dimensional analysis of doubly curved laminated shells. J Eng Mech. 1996;122(5):391401.

22. Wu CP, Lo JY. Three-dimensional elasticity solutions of laminated annular spherical shells. J Eng Mech. 2000;126(8):882-5.

23. Kumari P, Kar S. Static behavior of arbitrarily supported composite laminated cylindrical shell panels: an analytical 3D elasticity approach. Compos Struct. 2019;207:949-65.

24. Correia IFP, Soares CMM, Soares CAM, Herskovits J. Analysis of laminated conical shell structures using higher order models. Compos Struct. 2003;62(3):383-90.

25. Thakur SN, Ray C, Chakraborty S. A new efficient higher-order shear deformation theory for a doubly curved laminated composite shell. Acta Mech. 2017;228(1):69-87.

26. Shah PH, Batra RC. Stretching and bending deformations due to normal and shear tractions of doubly curved shells using third-order shear and normal deformable theory. Mech Adv Mater Struct. 2018;25(15-16):1276-96.

27. Dau F, Polit O, Touratier M. An efficient $C^{1}$ finite element with continuity requirements for multilayered/sandwich shell structures. Comput Struct. 2004;82(23):1889-99.

28. Yamamoto T, Yamada T, Matsui K. A quadrilateral shell element with degree of freedom to represent thickness-stretch. Comput Mech. 2017;59(4):625-46.

29. Paccola RR, Sampaio MSM, Coda HB. Continuous stress distribution following transverse direction for fem orthotropic laminated plates and shells. Appl Math Model. 2016;40(15):7382-409.

30. Sze KY, Yao LQ, Pian THH. An eighteen-node hybrid-stress solid-shell element for homogenous and laminated structures. Finite Elem Anal Des. 2002;38(4):353-74.

31. Fiolka M, Matzenmiller A. On the resolution of transverse stresses in solid-shells with a multi-layer formulation. Commun Numer Methods Eng. 2007;23(4):313-26.

32. Shiri S, Naceur H. Analysis of thin composite structures using an efficient hex-shell finite element. J Mech Sci Technol. 2013:27(12):3755-63.

33. Rah K, Van Paepegem W, Habraken AM, Degrieck J. A mixed solid-shell element for the analysis of laminated composites. Int J Numer Methods Eng. 2012;89(7):805-28.

34. Kwon YW. Analysis of laminated and sandwich composite structures using solid-like shell elements. Appl Compos Mater. 2013;20(4):355-73.

35. Kulikov GM, Plotnikova SV. Exact geometry four-node solid-shell element for stress analysis of functionally graded shell structures via advanced SaS formulation. Mech Adv Mater Struct. (In Press).

36. Jabareen M, Mtanes E. A solid-shell Cosserat point element for the analysis of geometrically linear and nonlinear laminated composite structures. Finite Elem Anal Des. 2018;142:61-80.

37. Leonetti L, Nguyen-Xuan H. A mixed edge-based smoothed solid-shell finite element method (MES-FEM) for laminated shell structures. Compos Struct. 2019;208:168-79.

38. Gol'denweizer AL. Theory of thin elastic shells., International series of monograph in aeronautics and astronauticsNew York: Pergamon Press; 1961.

39. Cicala P. Systematic approximation approach to linear shell theory. Torino: Levrotto e Bella; 1965.

40. Wu CP, Tarn JQ, Chen PY. Refined asymptotic theory of doubly curved laminated shells. J Eng Mech. 1997;123(12):1238-46.

41. Chung SW, Hong SG. Pseudo-membrane shell theory of hybrid anisotropic materials. Compos Struct. 2017;160:58693.

42. Reddy JN, Liu CF. A higher-order shear deformation theory of laminated elastic shells. Int J Eng Sci. 1985;23(3):319-30,

43. Jing HS, Tzeng KG. Analysis of thick laminated anisotropic cylindrical shells using a refined shell theory. Int J Solids Struct. 1995;32(10):1459-76.

44. Desai P, Kant T. On numerical analysis of axisymmetric thick circular cylindrical shells based on higher order shell theories by segmentation method. J Sandw Struct Mater. 2015;17(2):130-69.

45. Endo M. An alternative first-order shear deformation concept and its application to beam, plate and cylindrical shell models. Compos Struct. 2016;146:50-61.

46. Shah PH, Batra RC. Stress singularities and transverse stresses near edges of doubly curved laminated shells using tsndt and stress recovery scheme. Eur J Mech A/Solids. 2017;63:68-83.

47. Katili I, Maknun IJ, Batoz JL, Ibrahimbegovic A. Shear deformable shell element DKMQ24 for composite structures. Compos Struct. 2018;160:586-93.

48. Thakur SN, Ray C, Chakraborty S. Response sensitivity analysis of laminated composite shells based on higher-order shear deformation theory. Arch Appl Mech. 2018;88(8):1429-59.

49. Reinoso J, Paggi M, Areias P, Blázquez A. Surface-based and solid shell formulations of the 7-parameter shell model for layered CFRP and functionally graded power-based composite structures. Mech Adv Mater Struct. (in press)

50. Chaudhuri RA. On the prediction of interlaminar shear stresses in a thick laminated general shell. Int J Solids Struct. 1990;26(5):499-510.

51. He LH. A linear theory of laminated shells accounting for continuity of displacements and transverse shear stresses at layer interfaces. Int J Solids Struct. 1994;31(5):613-27.

52. Gruttmann F, Wagner W, Knust G. A coupled global-local shell model with continuous interlaminar shear stresses. Comput Mech. 2016;57(2):237-55.

53. Tahani M, Andakhshideh A, Maleki S. Interlaminar stresses in thick cylindrical shell with arbitrary laminations and boundary conditions under transverse loads. Compos Part B Eng. 2016;98:151-65.

54. Gruttman F, Knust G, Wagner W. Theory and numerics of layered shells with variationally embedded interlaminar stresses. Comput Methods Appl Mech Eng. 2017;326:713-38.

55. Knust G, Gruttmann F. A layered shell element for the computation of interlaminar shear stresses and thickness normal stresses. PAMM. 2017;17(1):323-4

56. Gruttmann F, Wagner W. Shear correction factors for layered plates and shells. Comput Mech. 2017;59(1):129-46. 
57. Brank B. On composite shell models with a piecewise linear warping function. Compos Struct. 2003;59(2):163-71.

58. Kumar A, Chakrabarti A, Bhargava P. Finite element analysis of laminated composite and sandwich shells using higher order zigzag theory. Compos Struct. 2013;106:270-81

59. Kumar A, Chakrabarti A, Bhargava P, Prakash V. Efficient failure analysis of laminated composites and sandwich cylindrical shells based on higher-order zigzag theory. J Aerosp Eng. 2015;28(4):04014100.

60. Coda HB, Paccola RR, Carrazedo R. Zig-Zag effect without degrees of freedom in linear and non linear analysis of laminated plates and shells. Compos Struct. 2017;161:32-50.

61. Ahmed A, Kapuria S. A four-node facet shell element for laminated shells based on the third order zigzag theory. Compos Struct. 2016;158(1):112-27.

62. Miri AK, Nosier A. Out-of-plane stresses in composite shell panels: layerwise and elasticity solutions. Acta Mech. 2011;220(1):15-32.

63. Naumenko K, Eremeyev VA. A layer-wise theory of shallow shells with thin soft core for laminated glass and photovoltaic applications. Compos Struct. 2017;178:434-46.

64. Ahmadi I. Interlaminar stress analysis in general thick composite cylinder subjected to nonuniform distributed radial pressure. Mech Adv Mater Struct. 2017;24(9):773-88.

65. Vidal P, Gallimard L, Polit O. Multiresolution strategies for the modeling of composite shell structures based on the variable separation method. Int J Numer Methods Eng. 2019;117(7):778-99.

66. Zenkour AM. Global structural behaviour of thin and moderately thick monoclinic spherical shells using a mixed shear deformation model. Arch Appl Mech. 2004;74(3):262-76.

67. Zucco G, Groh RMJ, Madeo A, Weaver PM. Mixed shell element for static and buckling analysis of variable angle tow composite plates. Compos Struct. 2016;152:324-38.

68. Cinefra M, Chinosi C, Della Croce L, Carrera E. Refined shell finite elements based on RMVT and MITC for the analysis of laminated structures. Compos Struct. 2014;113:492-7.

69. Prulière E. $3 d$ simulation of laminated shell structures using the proper generalized decomposition. Compos Struct. 2014;117:373-81. https://doi.org/10.1016/j.compstruct.2014.06.039.

70. Bognet B, Leygue A, Chinesta F. Separated representations of $3 d$ elastic solutions in shell geometries. Adv Model Simul Eng Sci. 2014;1 (1):4. https://doi.org/10.1186/2213-7467-1-4.

71. Carrera E. Historical review of zig-zag theories for multilayered plates and shells. Appl Mech Rev. 2003;56:287-308.

72. Carrera E, Cinefra M, Petrolo M, Zappino E. Finite element analysis of structures through unified formulation. Chichester: Wiley; 2014

73. Carrera E, Petrolo M. Guidelines and recommendation to construct theories for metallic and composite plates. AIAA J. 2010;48(12):2852-66.

74. Carrera E, Petrolo M. On the effectiveness of higher-order terms in refined beam theories. J Appl Mech. 2011; https:// doi.org/10.1115/1.4002207.

75. Carrera E, Cinefra M, Lamberti A, Petrolo M. Results on best theories for metallic and laminated shells including layer-wise models. Compos Struct. 2015;126:285-98.

76. Petrolo M, Carrera E. Best theory diagrams for multilayered structures via shell finite elements. Adv Model Simul Eng Sci. 2019;6(4):1-23.

77. Cheng B, Titterington DM. Neural networks: a review from a statistical perspective. Stat Sci. 1994;9(1):2-30. https:// doi.org/10.1214/ss/1177010638.

78. Kadi HE. Modeling the mechanical behavior of fiber-reinforced polymeric composite materials using artificial neural networks—a review. Compos Struct. 2006;73(1):1-23. https://doi.org/10.1016/j.compstruct.2005.01.020.

79. Worden K, Staszewski WJ, Hensman JJ. Natural computing for mechanical systems research: a tutorial overview. Mech Syst Signal Process. 2011;25(1):4-111. https://doi.org/10.1016/j.ymssp.2010.07.013.

80. Nasiri S, Khosravani MR, Weinberg K. Fracture mechanics and mechanical fault detection by artificial intelligence methods: a review. Eng Fail Anal. 2017;81:270-93. https://doi.org/10.1016/j.engfailanal.2017.07.011.

81. Balokas G, Czichon S, Rolfes R. Neural network assisted multiscale analysis for the elastic properties prediction of 3D braided composites under uncertainty. Compos Struct. 2018;183:550-62. https://doi.org/10.1016/j.compstruct.2017. 06.037 In honor of Prof. Y. Narita.

82. Koide RM, Ferreira APCS, Luersen MA. Laminated composites buckling analysis using lamination parameters, neural networks and support vector regression. Lat Am J Solids Struct. 2015;12:271-94.

83. Mallela UK, Upadhyay A. Buckling load prediction of laminated composite stiffened panels subjected to in-plane shear using artificial neural networks. Thin-Walled Struct. 2016;102:158-64. https://doi.org/10.1016/j.tws.2016.01.025.

84. Kumar CS, Arumugam V, Sengottuvelusamy R, Srinivasan S, Dhakal HN. Failure strength prediction of glass/epoxy composite laminates from acoustic emission parameters using artificial neural network. Appl Acoust. 2017;115:32-41. https://doi.org/10.1016/j.apacoust.2016.08.013.

85. Vineela MG, Dave A, Chaganti PK. Artificial neural network based prediction of tensile strength of hybrid composites. Mater Today Proc. 2018;5(9, Part 3):19908-15. https://doi.org/10.1016/j.matpr.2018.06.356 Materials Processing and characterization, 16th - 18th March 2018

86. Dey S, Naskar S, Mukhopadhyay T, Gohs U, Spickenheuer A, Bittrich L, Sriramula S, Adhikari S, Heinrich G. Uncertain natural frequency analysis of composite plates including effect of noise-a polynomial neural network approach. Compos Struct. 2016;143:130-42. https://doi.org/10.1016/j.compstruct.2016.02.007.

87. Dey S, Mukhopadhyay T, Spickenheuer A, Gohs U, Adhikari S. Uncertainty quantification in natural frequency of composite plates_-an artificial neural network based approach. Adv Compos Lett. 2016;25(2):096369351602500203. https://doi.org/10.1177/096369351602500203.

88. Karsh PK, Mukhopadhyay T, Dey S. Stochastic dynamic analysis of twisted functionally graded plates. Compos Part B Eng. 2018;147:259-78. https://doi.org/10.1016/j.compositesb.2018.03.043.

89. Gajewski J, Golewski P, Sadowski T. Geometry optimization of a thin-walled element for an air structure using hybrid system integrating artificial neural network and finite element method. Compos Struct. 2017;159:589-99. https://doi. org/10.1016/j.compstruct.2016.10.007. 
90. Bathe KJ, Dvorkin EN. A formulation of general shell elements-the use of mixed interpolation of tensorial components. Int J Numer Methods Eng. 1986;22(3):697-722.

91. Hagan MT, Demuth HB, Beale MH, De Jesús O. Neural network design. Oklahoma: Martin Hagan; 2014.

92. Krizhevsky A, Sutskever I, Hinton GE. Imagenet classification with deep convolutional neural networks. Commun ACM. 2017;60(6):84-90. https://doi.org/10.1145/3065386.

93. Cinefra M, Valvano S. A variable kinematic doubly-curved MITC9 shell element for the analysis of laminated composites. Mech Adv Mater Struct. 2016;23(11):1312-25.

94. Huang NN. Influence of shear correction factors in the higher-order shear deformation laminated shell theory. Int J Solids Struct. 1994;31:1263-77.

\section{Publisher's Note}

Springer Nature remains neutral with regard to jurisdictional claims in published maps and institutional affiliations.

\section{Submit your manuscript to a SpringerOpen ${ }^{\circ}$} journal and benefit from:

- Convenient online submission

- Rigorous peer review

- Open access: articles freely available online

- High visibility within the field

Retaining the copyright to your article

Submit your next manuscript at springeropen.com 